\title{
INTEGER REPRESENTATIONS OF CONVEX POLYGON INTERSECTION GRAPHS*
}

\author{
TOBIAS MÜLLER ${ }^{\dagger}$, ERIK JAN VAN LEEUWEN ${ }^{\ddagger}$, AND JAN VAN LEEUWEN $\$$
}

\begin{abstract}
We determine tight bounds on the smallest-size integer grid needed to represent the $n$-node intersection graphs of a convex polygon $P$ with $\mathrm{P}$ given in rational coordinates. The intersection graphs use only polygons that are geometrically similar to $P$ (translates or homothets) and must be represented such that each corner of each polygon lies on a point of the grid. We show the following generic results: if $P$ is a parallelogram and only translates of $P$ are used, then an $\Omega\left(n^{2}\right) \times \Omega\left(n^{2}\right)$ grid is sufficient and is needed for some graphs; if $P$ is any other convex polygon and only translates of $P$ are used, then a $2^{\Omega(n)} \times 2^{\Omega(n)}$ grid is sufficient and is needed for some graphs; if $P$ is any convex polygon and arbitrary homothets of $P$ are allowed, then a $2^{\Omega(n)} \times 2^{\Omega(n)}$ grid is sufficient and is needed for some graphs. The results substantially improve earlier bounds and settle the complexity of representing convex polygon intersection graphs. The results also imply small polynomial certificates for the recognition problem for all graph classes considered.
\end{abstract}

Key words. polygon intersection graphs, geometric representations of graphs, graph drawing

AMS subject classifications. 05C62, 68R 10

DOI. $10.1137 / 110825224$

1. Introduction. Intersection graphs are widely used for modeling purposes [10]. Many variants of these graphs have been studied, from a graph-theoretic and a computational perspective (see, e.g., $[20,29]$ ). In this paper we consider convex polygon intersection graphs, which arise naturally in planning and layout problems in the plane. We analyze the representation problem for these graphs and determine the complexity of representing them as geometric structures in computer memory. In particular, we will prove tight upper and lower bounds on the value of $N=2^{b(n)}$ such that any $n$-node convex polygon intersection graph can be realized with each corner of each polygon lying on a point of the $N \times N$ grid. The precise definitions will be given below. We will see that the bounds depend nontrivially on the underlying base polygon of the graphs.

We first define intersection graphs more precisely. Let $\mathcal{A}=\left\{A_{i}: i \in I\right\}$ be any collection of sets or objects. The intersection graph of $\mathcal{A}$ is a graph $G=(I, E)$ with vertex set $I$ and edge $i j \in E$ if and only if $A_{i} \cap A_{j} \neq \emptyset$. If $\mathcal{A}$ has $G$ as its intersection graph, then we say that $\mathcal{A}$ realizes $G$. By constraining the allowed collections $\mathcal{A}$ one obtains different classes of intersection graphs. For each class the following questions arise [29]: the recognition problem ("determine whether a given graph is realized as a member of the given class") and the representation problem ("represent a graph as an intersection graph of a given class"). Given an $n$-node intersection graph $G$, we typically want a realization of $G$ by an allowed $n$-element collection $\mathcal{A}$ that gives a representation in a minimum amount of memory space.

\footnotetext{
*Received by the editors February 22, 2011; accepted for publication (in revised form) November 30, 2012; published electronically January 31, 2013.

http://www.siam.org/journals/sidma/27-1/82522.html

$\dagger$ Mathematical Institute, Utrecht University, NL-3508 TA Utrecht, The Netherlands (t.muller@ uu.nl). This author's research was partially supported by a VENI grant from the Netherlands Organisation for Scientific Research (NWO).

${ }_{\ddagger}$ Department of Computer, Control, and Management Engineering, Sapienza University of Rome, I-00185 Roma, Italy (E.J.van.Leeuwen@dis.uniroma1.it).

$\S$ Department of Information and Computing Sciences, Utrecht University, NL-3508 TB Utrecht, The Netherlands (j.vanleeuwen@cs.uu.nl).
} 
The realization of graphs on $N \times N$ grids is well-motivated by the practical interest in drawing or embedding graphs in the plane on a two-dimensional pixel grid [23]. There is a considerable history of results on the smallest-size integer grid needed for various kinds of drawings of graphs, beginning with the seminal work by de Fraysseix, Pach, and Pollack [8] and Schnyder [27] for straight-line drawings of planar graphs. The grid model is common for many kinds of geometric intersection graphs as well; see, e.g., [7]. It is the ideal reference framework to use for convex polygon intersection graphs as well.

Convex polygon intersection graphs are obtained when the objects $A_{i} \in \mathcal{A}$ are all geometrically similar (either by translation or by general homothetic transformation) to a given convex polygon $P$. The graphs belong to the general family of geometric intersection graphs, in which the $A_{i} \in \mathcal{A}$ are all geometric objects in the plane. These graphs have been studied, e.g., for segments [16], continuous curves or "strings" [9, 14], unit disks $[4,7]$, isosceles right triangles or semisquares [13], and arbitrary convex sets $[24,26]$. The corresponding classes have a long history; see, for instance, the surveys in $[29,30]$.

Convex polygon intersection graphs generalize a variety of interesting classes such as triangle-intersection graphs [21], rectangle- or box-intersection graphs [25], and parallelogram- and trapezoid-intersection graphs [22], although these classes may allow nonhomothetic copies of the used polygons as well. The study of homothetic convex polygon intersection graphs in genere was initiated by Kratochvíl and Pergel [17], who proved that the recognition problem for these graphs is always NP-hard. However, the representation problem for these graphs was left open. In this paper we settle this problem by discrete geometrical arguments.

The representation problem has been studied for many other geometric intersection graphs, including segment graphs [16, 19], string graphs [15], (unit) disk graphs [19], and general convex set intersection graphs [24, 26]. The representations often need large grid sizes. For example, there exist segment, resp., (unit) disk, graphs that require a doubly exponential grid size if all endpoints, resp., centers and radii of the disks must be integer $[16,19]$. We will prove that for convex polygon intersection graphs, singly exponential-size grids are sufficient.

1.1. Problem description. In more precise terms, convex polygon intersection graphs are the intersection graphs of collections $\mathcal{A}=\left\{A_{i}: i \in I\right\}$ in which all $A_{i}$ are either translates or homothets of a fixed convex polygon $P$ in the plane. (A homothet of $P$ is any scaled and translated copy of $P$.) We call the intersection graph of a set of translates of $P$ a $P$-translate graph and the intersection graph of a set of homothets of $P$ a $P$-homothets graph.

Definition 1. Let $\operatorname{trans}(P)$ denote the set of all $P$-translate graphs and let $\operatorname{hom}(P)$ denote the set of all $P$-homothets graphs. Let $\operatorname{trans}_{n}(P), \operatorname{resp}, \operatorname{hom}_{n}(P)$, denote the set of all translate, resp., homothets, graphs on $n$ vertices.

In the representation problem for $P$-translate graphs and $P$-homothets graphs, we assume that all corner points of $P$ are given effectively, i.e., in rational coordinates. In this case it can be shown that for every $G \in \operatorname{trans}(P)$ there always is a collection $\mathcal{A}=\{A(v): v \in V(G)\}$ where each set is of the form $A(v)=p(v)+\lambda P$ with $\lambda \geq 0$, such that $\mathcal{A}$ realizes $G$ and all corners of $A(v)$ lie on $\mathbb{Z}^{2}$. (See, e.g., [31] or the proof of Theorem 2 below.) We call such an $\mathcal{A}$ an integer translate realization of $G$.

Likewise, it can be shown in this case that for every $G \in \operatorname{hom}(P)$ there always is a collection of $P$-homothets $\mathcal{A}=\{A(v): v \in V(G)\}$ such that $\mathcal{A}$ realizes $G$ and, moreover, every corner of every polygon of $\mathcal{A}$ lies on the integer grid $\mathbb{Z}^{2}$. (Again this 
will be proved formally in Theorem 2 below.) We call such an $\mathcal{A}$ an integer homothet realization of $G$.

The representation problem for $P$-translate and $P$-homothets graphs $G$ can now be defined as the problem of determining the smallest portion of the integer grid such that the translates, resp., homothets, of $P$ used in the realization of $G$ have all their corner points lying on this portion.

If $P$-translate or $P$-homothets graphs $G$ with $n$ nodes can be drawn on an integer $N \times N$ grid for $N=2^{b(n)}$, then they can be realized such that every corner point in the realization is fully specified by integer coordinates of at most $b(n)$ bits. Encoding their drawing on a grid, the graphs admit certificates of $\mathcal{O}(b(n) n)$ bits. (It is easy to verify whether a certificate encodes a $P$-translate or a $P$-homothets graph, respectively.) Thus, $b(n)$ is a crucial measure for the representation. Also note that polynomial-size certificates imply that the recognition problems are in NP [2].

1.2. Our results. To state our results formally, we introduce the following notation. If $\mathcal{A}$ is an integer translate or homothets realization of $G$, then

$$
m(\mathcal{A}):=\min \left\{K \in \mathbb{N}: A \subseteq[-K, K]^{2} \text { for all } A \in \mathcal{A}\right\},
$$

giving the biggest absolute value of a coordinate of a corner point of any element of $\mathcal{A}$. Next, for $G \in \operatorname{trans}(P)$, resp., $G \in \operatorname{hom}(P)$, we set

$$
t_{P}(G):=\min _{\mathcal{A}} m(\mathcal{A}), \quad h_{P}(G):=\min _{\mathcal{A}} m(\mathcal{A}),
$$

where the minimum is taken over all integer homothet, resp., translate, realizations $\mathcal{A}$ of $G$. Finally, we define

$$
t_{P}(n)=\max _{G \in \operatorname{trans}_{n}(P)} t_{P}(G), \quad h_{P}(n)=\max _{G \in \operatorname{hom}_{n}(P)} h_{P}(G)
$$

We prove precise upper and lower bounds on $t_{P}(n)$ and $h_{P}(n)$, thus settling the complexity of representing $P$-translate and $P$-homothets graphs, respectively. First we prove the following remarkable result for $t_{P}(n)$.

THEOREM 1. Let $P$ be a convex polygon with rational corner points. Then the following hold for $t_{P}(n)$ :

(i) If $P$ is a parallelogram, then $t_{P}(n)=\Theta\left(n^{2}\right)$.

(ii) If $P$ is not a parallelogram, then $t_{P}(n)=2^{\Theta(n)}$.

For homothets, on the other hand, there appears to be no qualitative difference between different kinds of convex polygon. We will prove the following.

THEOREM 2. Let $P$ be any convex polygon with rational corner points. Then $h_{P}(n)=2^{\Theta(n)}$.

Part (i) of Theorem 1 is a considerable improvement over a result of Czyzowicz et al. [7], who only showed that $t_{U}(n) \leq 2^{n-1}$, where $U$ denotes the unit square. Theorem 2 greatly improves a recent bound in [31], where it was shown that $h_{P}(n)=$ $2^{O\left(n^{4}\right)}$ for convex polygons with rational corner points.

Theorems 1 and 2 also show that $n$-node $P$-translate, resp., $P$-homothets, graphs can be represented using only $O\left(n^{2}\right)$ bits $(O(n)$ per vertex), when $P$ has rational corner points. It follows that $\operatorname{trans}(P)$ and $\operatorname{hom}(P)$ have small polynomial-size certificates, hence recognition problems in NP, for all convex polygons $P$ with rational corner points. This improves on earlier results about the recognition of these graphs [24, 31]. 
The latter applies to the recognition problem of seemingly different classes as well, like max-tolerance graphs (see [11]). Kaufmann et al. [13] showed that this class coincides with the class of intersection graphs of "semisquares," i.e., isosceles right triangles. By Lemma 1 below, the class of intersection graphs of scaled, translated copies of $P$ is the same for all triangles $P$. As Kaufmann et al. [13] proved that max-tolerance graph recognition is NP-hard and Theorem 2 implies polynomial-size certificates and thus that the problem is in NP, it follows that the recognition problem for max-tolerance graphs is NP-complete.

1.3. Organization of the paper. The upper bounds are proved in section 2 . The lower bounds for $P$-translates are shown in section 3 for the case when $P$ is a parallelogram and in section 4 when $P$ is not. The lower bounds for $P$-homothets are shown in section 5 . In section 6 we present some possible directions for further work.

1.4. Notation. We use the following notation throughout. We write $P(x): Q(x)$ to denote (the set of) all entities $P(x)$, where $x$ satisfies property $Q(x)$. For points $p \in \mathbb{R}^{2}$ we denote by $p_{x}$ its $x$-coordinate and by $p_{y}$ its $y$-coordinate. If $x, y$ are two vectors of the same length, then $d_{\mathrm{H}}(x, y)$ denotes the Hamming distance of vectors $x, y$, i.e., the number of coordinates in which the vectors differ. For sets $A \subseteq \mathbb{R}^{2}, \operatorname{cl}(A)$ denotes their topological closure. For sets $A, B$ we let $A+B=\{p+q: p \in A, q \in B\}$ (the Minkowski sum). Given a graph $G=(V, E)$ the closed neighborhood of a vertex $v \in V$ is $\bar{N}(v):=\{v\} \cup\{u: u v \in E\}$.

\section{Upper bounds.}

2.1. Upper bound for translates of a parallelogram. In this section we shall prove that if $P$ is a parallelogram with rational corner points, then $t_{P}(n)=$ $O\left(n^{2}\right)$.

Recall that an affine transformation is a nonsingular linear map followed by a translation.

LEMma 1. Let $P, Q$ be two polygons. If there is an affine transformation $T$ such that $P=T[Q]$, then $\operatorname{hom}(P)=\operatorname{hom}(Q)$ and $\operatorname{trans}(P)=\operatorname{trans}(Q)$.

Proof. Observe that for all $x, y \in \mathbb{R}^{2}$ and $\lambda, \mu>0$, we have

$$
(x+\lambda Q) \cap(y+\mu Q) \neq \emptyset \Leftrightarrow(T x+\lambda T[Q]) \cap(T y+\mu T[Q])=\emptyset .
$$

This immediately implies that every $Q$ homothets (resp., translate) graph is also a $P$ homothets (resp., translate) graph. Since the inverse of an affine transformation is again an affine transformation, the same reasoning applied to $P$ and $T^{-1}$ also gives that every $P$ homothets (resp., translate) graph is also a $Q$ homothets (resp., translate) graph.

Lemma 2. Let $P, Q$ be two polygons whose corner points have rational coordinates. If there is an affine transformation $T$ such that $P=T[Q]$, then $t_{P}(n)=\Theta\left(t_{Q}(n)\right)$ and $h_{P}(n)=\Theta\left(h_{Q}(n)\right)$.

Proof. We first claim that there is a nonsingular integer matrix $U \in \mathbb{Z}^{2 \times 2}$ such that $U[Q]$ is a homothet $p+\lambda P$ of $P$.

To see this, let $c_{1}, c_{2}, c_{3} \in \mathbb{Q}^{2}$ be three distinct corners of $Q$. Observe that $T\left(c_{1}\right), T\left(c_{2}\right), T\left(c_{3}\right)$ are distinct corners of $P$. In particular $T\left(c_{1}\right), T\left(c_{2}\right), T\left(c_{3}\right) \in \mathbb{Q}^{2}$. Because $c_{1}, c_{2}, c_{3}$ are distinct corner points, it must be that $v_{1}:=c_{2}-c_{1}, v_{2}:=c_{3}-c_{1}$ are linearly independent. Being an affine transformation, $T$ is of the form $T z=S z+b$ for some nonsingular matrix $S$. We have $T\left(c_{2}\right)-T\left(c_{1}\right)=S\left(v_{1}\right) \in \mathbb{Q}^{2}$ and similarly $S\left(v_{2}\right) \in \mathbb{Q}^{2}$. Since $v_{1}, v_{2}$ are rational and linearly independent, there are $\lambda_{1}, \lambda_{2} \in \mathbb{Q}$ 
such that $e_{1}=\lambda_{1} v_{2}+\lambda_{2} v_{2}$, where $e_{1}=(1,0)^{T}$ as usual. It follows that $S\left(e_{1}\right)$, the first column of $S$ is rational. Similarly the second column of $S$ is rational. Let $\lambda$ be the product of the denominators of all entries of $S$ and set $U:=\lambda \cdot S$. (If an entry happens to be zero, then we take one to be its denominator.) Then clearly $U \in \mathbb{Z}^{2 \times 2}$. Observe that $U[Q]=\lambda \cdot(T[Q]-b)=(-\lambda) b+\lambda P$ is a homothet of $P$ as required. This proves the claim (with $p:=-\lambda b$ ).

Now suppose that a graph $G \in \operatorname{hom}_{n}(Q)=\operatorname{hom}_{n}(P)$ is realized by the $Q$ homothets $A_{1}=p_{1}+\lambda_{1} Q, \ldots, A_{n}=p_{1}+\lambda_{n} Q$ with integer corner points. Observe that $A_{i} \cap A_{j} \neq \emptyset$ if and only if $U\left[A_{i}\right] \cap U\left[A_{j}\right] \neq \emptyset$ for all $i, j$ (as $U$ is nonsingular). Thus $U\left[A_{1}\right], \ldots, U\left[A_{n}\right]$ also represent $G$. Observe that for each $i$ we have that $U\left[A_{i}\right]=$ $U\left[p_{i}\right]+\lambda_{i} p+\lambda_{i} \lambda P$ is a homothet of $P$. Moreover, since the corner points of $A_{i}$ lie on the integer grid and $U \in \mathbb{Z}^{2 \times 2}$, the corner points of $U\left[A_{i}\right]$ also lie on the integer grid. Now observe that if $A_{1}, \ldots, A_{n} \subseteq[-K, K]^{2}$, then $U\left[A_{1}\right], \ldots, U\left[A_{n}\right] \subseteq[-L, L]^{2}$, where $L=K \cdot \max _{1 \leq i, j \leq 2}\left|U_{i j}\right|$. This shows that $h_{P}(n)=O\left(h_{Q}(n)\right.$ ) and (by taking $\lambda_{1}=\cdots=\lambda_{n}$ identical) also that $t_{P}(n)=O\left(t_{Q}(n)\right)$.

Since $T^{-1}$ is also a linear transformation, and $Q=T^{-1}[P]$, the same proof also gives that $h_{Q}(n)=O\left(h_{P}(n)\right)$ and $t_{Q}(n)=O\left(t_{P}(n)\right)$. The concludes the proof of the lemma.

Corollary 1. Let $P$ be a parallelogram with rational corner points, and let $U$ denote the unit square. Then $t_{P}(n)=\Theta\left(t_{U}(n)\right)$.

THEOREM 3. If $P$ is a parallelogram with rational corner points, then $t_{P}(n)=$ $O\left(n^{2}\right)$.

Proof. By Corollary 1 we can restrict attention to the case when $P=U$. In that case, there are unit intervals $I_{1}, \ldots, I_{n}$ and $J_{1}, \ldots, J_{n}$ such that $i j \in E$ if and only if $\left(I_{i} \times J_{i}\right) \cap\left(I_{j} \times J_{j}\right) \neq \emptyset$. In other words $i j \in E$ if and only if both $I_{i} \cap I_{j} \neq \emptyset$ and $J_{i} \cap J_{j} \neq \emptyset$. Let $G_{1}=\left(V, E_{1}\right)$ denote the intersection graph defined by the intervals $I_{1}, \ldots, I_{n}$ and let $G_{2}=\left(V, E_{2}\right)$ denote the intersection graph defined by $J_{1}, \ldots, J_{n}$. (Thus, $i j \in E_{1}$ if and only if $I_{i} \cap I_{j} \neq \emptyset$ and similarly for $E_{2}$.)

According to a result of Corneil et al. [6] every unit interval graph on $n$ vertices has a representation with unit intervals whose endpoints are multiples of $\frac{1}{n}$. Hence, we can assume that the endpoints of the $I_{i}$ 's and $J_{i}$ 's are multiples of $\frac{1}{n}$. Notice that for any component of $G_{1}$ of order $k$, the corresponding intervals $I_{i_{1}}, \ldots, I_{i_{k}}$ are contained in an interval of length at most $k$. By performing componentwise translations if needed we can also assume that all intervals $I_{i}$ are contained in $\left[0, n+\frac{n-1}{n}\right]$ (i.e., we order the components of $G_{1}$ arbitrarily, we simultaneously translate all intervals of the first component so that 0 is the leftmost endpoint, then we simultaneously translate all intervals of the second component so that its leftmost endpoint is $\frac{1}{n}$ to the right of the rightmost endpoint of the first component, and so on). Similarly we can assume that $J_{1}, \ldots, J_{n} \subseteq\left[0, n+\frac{n-1}{n}\right]$.

Finally, we scale the whole construction by $n$ (i.e., we replace $I_{i}$ by $I_{i}^{\prime}=n \cdot I_{i}$ and $J_{i}$ by $\left.J_{i}^{\prime}:=n \cdot J_{i}\right)$. This gives a representation of $G$ by same-size squares in which all coordinates of the corner points are integers $\in\left\{0, \ldots, n^{2}+n-1\right\}$.

2.2. Upper bound for translates and homothets. We will now prove that if $P$ is any convex polygon with rational corner points, then $t_{P}(n)=2^{O(n)}$ and $h_{P}(n)=2^{O(n)}$.

We will use the following observation.

Lemma 3. Let $P$ be a convex polygon with rational coordinates. Then there exists an $N=N(P) \in \mathbb{N}$ and $N$ vectors $C_{i}=C_{i}(P)=\left(a_{i}, b_{i}, c_{i}, d_{i}, e_{i}, f_{i}\right) \in \mathbb{Q}^{6}$ such that 
$\left(\left(x_{1}, y_{1}\right)+\lambda_{1} P\right) \cap\left(\left(x_{2}, y_{2}\right)+\lambda_{2} P\right) \neq \emptyset$ if and only if $a_{i} x_{1}+b_{i} y_{1}+c_{i} \lambda_{1}+d_{i} x_{2}+e_{i} y_{2}+$ $f_{i} \lambda_{2} \geq 0$ for all $i=1, \ldots, N$.

Proof. We can write $P=\left\{x \in \mathbb{R}^{2}: A x \leq b\right\}$ for some matrix $A \in \mathbb{Q}^{k \times 2}$ and $b \in \mathbb{Q}^{k}$. Observe that $\left(p_{1}+\lambda_{1} P\right) \cap\left(p_{2}+\lambda_{2} P\right) \neq \emptyset$ if and only if there exists an $x \in \mathbb{R}^{2}$ such that

$$
A\left(\frac{x-p_{1}}{\lambda_{1}}\right) \leq b \text { and } A\left(\frac{x-p_{2}}{\lambda_{2}}\right) \leq b
$$

Or, in other words, $\left(p_{1}+\lambda_{1} P\right) \cap\left(p_{2}+\lambda_{2} P\right) \neq \emptyset$ if and only if there exists an $x \in \mathbb{R}^{2}$ such that $A^{\prime} x \leq b^{\prime}$, where we set

$$
A^{\prime}:=\left[\begin{array}{c}
A \\
A
\end{array}\right], \quad b^{\prime}:=\left(\begin{array}{c}
\lambda_{1} b+A p_{1} \\
\lambda_{2} b+A p_{2}
\end{array}\right)
$$

By a variant of Farkas' lemma (Corollary 7.1e in [28]) the existence of an $x \in \mathbb{R}^{2}$ such that $A^{\prime} x \leq b^{\prime}$ is equivalent to $y^{T} b^{\prime} \geq 0$ for all $y \in W$, where $W:=\left\{y \in \mathbb{R}^{2 k}: y \geq\right.$ $\left.0, y^{T} A^{\prime}=0\right\}$. Observe that we can write

$$
W=\left\{\mu_{1} y_{1}+\cdots+\mu_{N} y_{N}: \mu_{1}, \ldots, \mu_{N} \geq 0\right\},
$$

where $y_{1}, \ldots, y_{N} \in \mathbb{Q}^{2 k}$ are the vertices of the polytope $W^{\prime}:=\left\{y \in W: \sum y=1\right\}$. (That the $y_{i}$ 's have rational coordinates follows from the fact that all entries of $A^{\prime}$ are rational.) Then $y^{T} b^{\prime} \geq 0$ for all $y \in W$ if and only if $y_{i}^{T} b^{\prime} \geq 0$ for all $i=1, \ldots, N$. Finally, notice that by grouping terms together we can rewrite $y_{i}^{T} b^{\prime}$ as

$$
y_{i}^{T} b^{\prime}=a_{i} \cdot\left(p_{1}\right)_{x}+b_{i} \cdot\left(p_{1}\right)_{y}+c_{i} \lambda_{1}+d_{i} \cdot\left(p_{2}\right)_{x}+e_{i} \cdot\left(p_{2}\right)_{y}+f_{i} \lambda_{2}
$$

with $a_{i}, \ldots, f_{i} \in \mathbb{Q} . \quad \square$

THEOREM 4. Let $P$ be a convex polygon with rational corner points. Then $t_{P}(n)=2^{O(n)}$ and $h_{P}(n)=2^{O(n)}$.

Proof. By Lemma 2 we can assume without loss of generality that the corner points of $P$ lie on the integer grid. Let $C_{1}, \ldots, C_{N} \in \mathbb{Q}^{6}$ be as provided by Lemma 3 . (Recall that $C_{i}=\left(a_{i}, \ldots, f_{i}\right)$ and that $\left(\left(x_{1}, y_{1}\right)+\lambda_{1} P\right) \cap\left(\left(x_{2}, y_{2}\right)+\lambda_{2} P\right) \neq \emptyset$ if and only if $a_{i} x_{1}+b_{i} y_{1}+c_{i} \lambda_{1}+d_{i} x_{2}+e_{i} y_{2}+f_{i} \lambda_{2} \geq 0$ for all $i=1, \ldots, N$.) Let $G$ be a $P$-translate, resp., $P$-homothet, graph. For convenience we take $V(G)=\{1, \ldots, n\}$. We will say that $z=\left(x_{1}, y_{1}, \lambda_{1}, \ldots, x_{n}, y_{n}, \lambda_{n}\right) \in \mathbb{R}^{3 n}$ realizes $G$ as a translate, resp., homothet, graph if, when we set $P_{i}:=\left(x_{i}, y_{i}\right)^{T}+\lambda_{i} P$, the intersection graph of $P_{1}, \ldots, P_{n}$ is precisely $G$, where in the case of a translate representation we demand in addition that $\lambda_{1}=\cdots=\lambda_{n}$. Observe that there are representations with all the $x_{i}{ }^{\prime} \mathrm{s}, y_{i}{ }^{\prime}$ s, and $\lambda_{i}{ }^{\prime}$ 's nonnegative. Also observe that if $z$ realizes $G$ and $\mu>0$, then $\mu z=\left(\mu x_{1}, \ldots, \mu \lambda_{n}\right)$ also realizes $G$. Thus, there is a realization $z_{0}$ such that for each $1 \leq i \leq N$ and for each $1 \leq j_{1} \neq j_{2} \leq n$ we have either

$$
a_{i} x_{j_{1}}+b_{i} y_{j_{1}}+c_{i} \lambda_{j_{1}}+d_{i} x_{j_{2}}+e_{i} y_{j_{2}}+f_{i} \lambda_{j_{2}} \geq 0
$$

or

$$
a_{i} x_{j_{1}}+b_{i} y_{j_{1}}+c_{i} \lambda_{j_{1}}+d_{i} x_{j_{2}}+e_{i} y_{j_{2}}+f_{i} \lambda_{j_{2}} \leq-1
$$

(To find $z_{0}$, let $z$ be an arbitrary realization, and let us write $\varphi\left(j_{1}, j_{2}, i\right):=a_{i} x_{j_{1}}+$ $b_{i} y_{j_{1}}+c_{i} \lambda_{j_{1}}+d_{i} x_{j_{2}}+e_{i} y_{j_{2}}+f_{i} \lambda_{j_{2}}$. Then $\alpha:=\max \left\{\varphi\left(j_{1}, j_{2}, i\right): \varphi\left(j_{1}, j_{2}, i\right)<0\right\}$ is negative. Now set $z_{0}:=(1 /|\alpha|) \cdot z$.)

Copyright (c) by SIAM. Unauthorized reproduction of this article is prohibited. 
Consider the set of $N \cdot\left(\begin{array}{l}n \\ 2\end{array}\right)$ inequalities of this kind that $z_{0}$ satisfies. Let us write this set of inequalities as

$$
A z \leq b,
$$

where we also add the inequalities $x_{i}, y_{i}, \lambda_{i} \geq 0$ and in the translate graph case also the inequalities $\lambda_{i}=\lambda_{i+1}$ for $i=1, \ldots, n-1$. This way we obtain a system with $A \in \mathbb{Q}^{m \times 3 n}, b \in\{-1,0\}^{m}$, where $m=N\left(\begin{array}{l}n \\ 2\end{array}\right)+5 n-2$ in the translate case and $m=N\left(\begin{array}{l}n \\ 2\end{array}\right)+3 n$ in the homothet case.

The crucial observation for the proof is that if $z$ is another solution of (1), then $z$ also realizes $G$ as a homothets, resp., translate, graph. This follows from Lemma 3 and the fact that for each $1 \leq i \leq N, 1 \leq j_{1} \neq j_{2} \leq n$ the expression $a_{i} x_{j_{1}}+b_{i} y_{j_{1}}+$ $c_{i} \lambda_{1}+d_{i} x_{j_{2}}+e_{i} y_{j_{2}}+f_{i} \lambda_{2}$ is nonnegative for $z$ if and only if it is nonnegative for $z_{0}$.

The set $W:=\left\{z \in \mathbb{R}^{3 n}: A z \leq b\right\}$ is a polyhedron. Let $v$ be a vertex of $W$. Then $v=B^{-1} c$ is the unique solution to a system $B z=c$ that is obtained by taking $3 n$ of the inequalities of (1) and making them into equalities. By Cramer's rule

$$
v_{j}=\operatorname{det}\left(B_{j}\right) / \operatorname{det}(B),
$$

where $B_{j}$ is the matrix we get by replacing the $j$ th column of $B$ by $c$. Observe that all entries of $B$ and $B_{j}$ either are 0 or are elements of a finite set of rationals $Q=\left\{\frac{n_{1}}{d_{1}}, \ldots, \frac{n_{K}}{d_{K}}\right\}\left(Q\right.$ contains \pm 1 and \pm the coefficients of $\left.C_{1}, \ldots, C_{N}\right)$. Let us also observe that every row of $A$ (and hence $B$ ) has at most six nonzero elements, and hence every row of $B_{j}$ has at most seven nonzero elements.

Set

$$
\mu:=\left|\left(d_{1} \ldots d_{K}\right)^{3 n} \operatorname{det}(B)\right|, \quad w:=\mu v .
$$

Then $w$ realizes $G$ by a previous remark. Recall that by the determinant formula

$$
\operatorname{det}\left(B_{j}\right)=\sum_{\sigma}(-1)^{\sigma} \prod_{i=1}^{3 n}\left(B_{j}\right)_{i \sigma(i)}
$$

where the sum is over all permutations of $\{1, \ldots, 3 n\}$. Each nonzero summand of the right-hand side of $(2)$ is a rational number whose denominator divides $\left(d_{1} \ldots d_{K}\right)^{3 n}$ (since all entries of $B_{j}$ are elements of $Q$ ). Thus, we see that $w \in \mathbb{Z}^{3 n}$.

It remains to upper bound the entries of $w$. Recall that at most seven entries of each row of $B_{j}$ are nonzero. Hence in (2) there are at most $7^{3 n}$ summands that are not zero (corresponding to those permutations that map $i$ to a $\sigma(i)$ with $\left(B_{j}\right)_{i \sigma(i)}$ nonzero for all $i=1, \ldots, 3 n)$. Hence

$$
\left|w_{j}\right|=\left|\left(d_{1} \cdots d_{K}\right)^{3 n} \operatorname{det}\left(B_{j}\right)\right| \leq\left|\left(d_{1} \cdots d_{K}\right)^{3 n}\right| \cdot 7^{3 n} \cdot\left(\max _{q \in Q}|q|\right)^{3 n}=C^{n},
$$

writing $C:=\left|\left(d_{1} \cdots d_{K}\right)^{3}\right| \cdot 7^{3} \cdot \max _{q \in Q}\left|q^{3}\right|$. As remarked earlier, we can assume that $P$ has integer corner points. This implies that $w$ corresponds to a homothets, resp., translates, representation of $G$ with all corner points on integer points, and every coordinate of every corner point is $O\left(C^{n}\right)=2^{O(n)}$.

Theorem 4 considerably improves the bound given in [31]. We also note that Lemma 3 and Theorem 4 and their proofs generalize to higher dimensions. Thus, the proven bounds hold for the representation of $P$-translate and $P$-homothet graphs in any fixed, constant dimension greater than or equal to two and any convex polytope $P$ with rational corner points. 

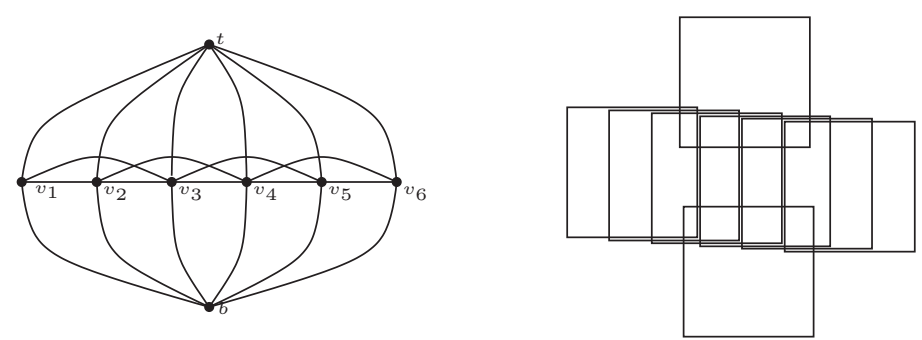

FIG. 1. The graph $\mathrm{O}_{3}$, and a unit square representation of it.

3. Lower bound for $\boldsymbol{P}$-translate graphs when $\boldsymbol{P}$ is a parallelogram. In this section we will prove that if $P$ is a parallelogram (with rational corner points), then $t_{P}(n)=\Omega\left(n^{2}\right)$. The result is obtained by constructing an infinite family of graphs $\left\{G_{n}: n \in \mathbb{N}\right\}$ that are all $U$-translate graphs and such that $G_{n}$ has $O(n)$ vertices and requires a grid of size $K=\Omega\left(n^{2}\right)$, where $U$ denotes the unit square. To construct the graphs $G_{n}$ we first define graphs $O_{n}$ and $L_{n}$.

For $n \geq 1$, we let $O_{n}$ be the graph with vertex set

$$
V\left(O_{n}\right):=\left\{b, t, v_{1}, \ldots, v_{2 n}\right\}
$$

and the following edge set:

$$
E\left(O_{n}\right):=\left\{v_{i} t, v_{i} b: i=1, \ldots, 2 n\right\} \cup\left\{v_{i} v_{j}:|i-j|<n, i \neq j\right\} .
$$

One easily notes that $O_{n}$ is a unit square translate graph for all $n$ (see Figure 1).

Let $U$ denote the unit square. Recall that for $p \in \mathbb{R}^{2}$, we denote its $x$-coordinate by $p_{x}$ and its $y$-coordinate by $p_{y}$.

Lemma 4. Let the scalar $\lambda \geq 0$, and let the points $\left\{p(v): v \in V\left(C_{4}\right)\right\}$ be such that if we set $P(v):=p(v)+\lambda U$ for each $v \in V\left(C_{4}\right)$, then the set of polygons $\{P(v)$ : $\left.v \in V\left(C_{4}\right)\right\}$ has $C_{4}$ as its intersection graph. Pick $u, v \in V\left(C_{4}\right)$ with uv $\notin E\left(C_{4}\right)$. Exactly one of $\left[(p(u))_{x},(p(u))_{x}+\lambda\right] \cap\left[(p(v))_{x},(p(v))_{x}+\lambda\right]$ and $\left[(p(u))_{y},(p(u))_{y}+\lambda\right] \cap$ $\left[(p(v))_{y},(p(v))_{y}+\lambda\right]$ is nonempty.

Proof. Since $P(u)$ and $P(v)$ do not intersect, at least one of the intersections $\left[(p(u))_{x},(p(u))_{x}+\lambda\right] \cap\left[(p(v))_{x},(p(v))_{x}+\lambda\right]$ and $\left[(p(u))_{y},(p(u))_{y}+\lambda\right] \cap\left[(p(v))_{y},(p(v))_{y}+\right.$ $\lambda]$ must be empty.

Aiming for a contradiction, let us suppose that both intersections are empty. We can assume that $(p(u))_{x}>(p(v))_{x}+\lambda$ and $(p(u))_{y}>(p(v))_{y}+\lambda$. (The other possibilities can be dealt with in a similar way.) Let $w, s$ be the two vertices of $C_{4}$ different from $u, v$. Observe that $w s \notin V\left(C_{4}\right)$. Since $w$ is adjacent to both $u$ and $v$, we must have

$$
\begin{aligned}
(p(w))_{x} & \in\left[(p(u))_{x}-\lambda,(p(u))_{x}+\lambda\right] \cap\left[(p(v))_{x}-\lambda,(p(v))_{x}+\lambda\right] \\
& \subseteq\left[(p(u))_{x}-\lambda,(p(v))_{x}+\lambda\right] \\
& \subseteq\left[(p(v))_{x},(p(v))_{x}+\lambda\right]
\end{aligned}
$$

where we have used that $(p(u))_{x}>(p(v))_{x}+\lambda$ in the last line. Completely analogously, we have $(p(w))_{y} \in\left[(p(v))_{y},(p(v))_{y}+\lambda\right]$, and by symmetry $(p(s))_{x} \in\left[(p(v))_{x},(p(v))_{x}+\right.$ $\lambda]$ and $(p(s))_{y} \in\left[(p(v))_{y},(p(v))_{y}+\lambda\right]$. But this implies that $\left|(p(w))_{x}-(p(s))_{x}\right|, \mid(p(w))_{y}-$ $(p(s))_{y} \mid \leq \lambda$. In other words, $P(w) \cap P(s) \neq \emptyset$, a contradiction.

Lemma 5. Suppose that the scalar $\lambda \in \mathbb{N}$ and the points $\left\{p(v) \in \mathbb{Z}^{2}: v \in V\left(O_{n}\right)\right\}$ are such that if we set $P(v)=p(v)+\lambda U$ for each $v \in V\left(O_{n}\right)$, then the set of polygons $\left\{P(v): v \in V\left(O_{n}\right)\right\}$ has $O_{n}$ as its intersection graph. Then $\lambda \geq n / 2$. 

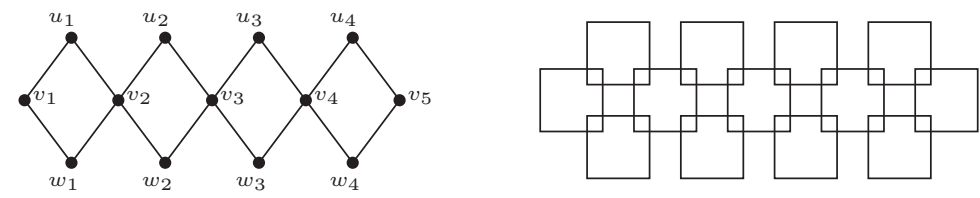

FIG. 2. The graph $L_{5}$, and a unit square representation of it.

Proof. Since $v_{1}, t, v_{2 n}, b$ is an induced copy of $C_{4}$ in $O_{n}$, appealing to Lemma 4 , we can assume without loss of generality that

$$
\begin{aligned}
& (p(b))_{x} \leq(p(t))_{x} \leq(p(b))_{x}+\lambda, \\
& (p(t))_{y}>(p(b))_{y}+\lambda .
\end{aligned}
$$

We must then have that $\left(p\left(v_{i}\right)\right)_{y} \in\left[(p(b))_{y},(p(b))_{y}+\lambda\right]$ for all $i=1, \ldots, 2 n$, since $v_{i}$ is adjacent to both $b$ and $t$. This implies that $\left|\left(p\left(v_{j}\right)\right)_{x}-\left(p\left(v_{j}\right)\right)_{x}\right| \leq \lambda$ if and only if $v_{i} v_{j} \in E\left(O_{n}\right)$. Since the closed neighborhoods $\bar{N}\left(v_{1}\right)=\left\{v_{1}, \ldots, v_{n}, t, b\right\}, \bar{N}\left(v_{2}\right)=$ $\left\{v_{1}, \ldots, v_{n+1}, t, b\right\}, \ldots, \bar{N}\left(v_{n+1}\right)=\left\{v_{2}, \ldots, v_{2 n}, t, b\right\}$ are all distinct, we must then also have that $\left(p\left(v_{1}\right)\right)_{x}, \ldots,\left(p\left(v_{n}\right)\right)_{x}$ are all distinct. Since $\left(p\left(v_{1}\right)\right)_{x}, \ldots,\left(p\left(v_{n}\right)\right)_{x} \subseteq$ $\left[(p(b))_{x}-\lambda,(p(b))_{x}+\lambda\right]$ (because $b v_{i}$ is an edge for all $i$ ), we must have that $2 \lambda+1 \geq$ $n+1$. Thus $\lambda \geq n / 2$ as required.

Next, we let $L_{n}$ denote the graph with vertex set

$$
V\left(L_{n}\right):=\left\{v_{1}, \ldots, v_{n}, u_{1}, \ldots, u_{n-1}, w_{1}, \ldots, w_{n-1}\right\}
$$

and the following edge set:

$$
E\left(L_{n}\right):=\left\{v_{i} u_{i}, v_{i} w_{i}, u_{i} v_{i+1}, w_{i} v_{i+1}: i=1, \ldots, n-1\right\} .
$$

Then $L_{n}$ is clearly a $P$-translate graph for all $n$ (see Figure 2).

Lemma 6. Suppose that the scalar $\lambda \geq 0$ and the points $\left\{p(v) \in \mathbb{R}^{2}: v \in V\left(L_{n}\right)\right\}$ are such that if we set $P(v)=p(v)+\lambda U$ for each $v \in V\left(L_{n}\right)$, then the set of polygons $\left\{P(v): v \in L_{n}\right\}$ has $L_{n}$ as its intersection graph. Then $\left\|p\left(v_{1}\right)-p\left(v_{n}\right)\right\| \geq \lambda(n-1)$.

Proof. Since $v_{1}, u_{1}, v_{2}, w_{1}$ is an induced copy of $C_{4}$ in $O_{n}$, appealing to Lemma 4 , we can assume without loss of generality that

$$
\begin{aligned}
& \left(p\left(u_{1}\right)\right)_{x} \leq\left(p\left(w_{1}\right)\right)_{x} \leq\left(p\left(u_{1}\right)\right)_{x}+\lambda \\
& \left(p\left(u_{1}\right)\right)_{y}>\left(p\left(w_{1}\right)\right)_{y}+\lambda .
\end{aligned}
$$

We must have $\left(p\left(v_{1}\right)\right)_{y},\left(p\left(v_{2}\right)\right)_{y} \in\left[\left(p\left(w_{1}\right)\right)_{y},\left(p\left(w_{1}\right)\right)_{y}+\lambda\right]$ and, appealing to Lemma 4, we can assume without loss of generality that $\left(p\left(v_{1}\right)\right)_{x}+\lambda<\left(p\left(v_{2}\right)\right)_{x}$. We shall use induction to prove that in fact

$$
\begin{aligned}
\left(p\left(v_{i}\right)\right)_{x}+\lambda & <\left(p\left(v_{i+1}\right)\right)_{x}, \\
\left(p\left(u_{i}\right)\right)_{x},\left(p\left(w_{i}\right)\right)_{x} & \in\left[\left(p\left(v_{i}\right)\right)_{x},\left(p\left(v_{i}\right)\right)_{x}+\lambda\right], \\
\left|\left(p\left(u_{i}\right)\right)_{y}-\left(p\left(w_{i}\right)\right)_{y}\right| & >\lambda
\end{aligned}
$$

for $i=1, \ldots, n-1$. This clearly implies the claim.

Clearly (4) is true when $i=1$. Suppose that (4) holds for some $i<n-$ 1. Observe that two same-size squares intersect if and only if they both contain one of the other's corner points. Hence $P\left(u_{i}\right), P\left(w_{i}\right), P\left(u_{i+1}\right), P\left(w_{i+1}\right)$ must each contain a different corner point of $P\left(v_{i+1}\right)$. Moreover, by the second line of (4), 
$P\left(u_{i}\right), P\left(w_{i}\right)$ must contain the top-left and bottom-left corners of $P\left(w_{i-1}\right)$. Hence $P\left(u_{i+1}\right), P\left(w_{i+1}\right)$ contain the top-right and bottom-right corners of $P\left(v_{i+1}\right)$. This gives $\left(p\left(u_{i+1}\right)\right)_{x},\left(p\left(w_{i+1}\right)\right)_{x} \in\left[\left(p\left(v_{i+1}\right)\right)_{x},\left(p\left(v_{i+1}\right)\right)_{x}+\lambda\right]$. Since $u_{i+1} w_{i+1} \notin E\left(L_{n}\right)$ we must then have $\left|\left(p\left(u_{i+1}\right)\right)_{y}-\left(p\left(w_{i+1}\right)\right)_{y}\right|>\lambda$. Let us assume that $\left(p\left(u_{i+1}\right)\right)_{y}>$ $\left(p\left(w_{i+1}\right)\right)_{y}+\lambda$ (the other case is similar). Since $v_{i+1}, v_{i+2}$ are both adjacent to $u_{i+1}, w_{i+1}$ we must have $\left.\left.\left(p\left(v_{i+1}\right)\right)_{y},\left(p\left(v_{i+2}\right)\right)_{y} \in\left[p\left(w_{i+1}\right)\right)_{y}, p\left(w_{i+1}\right)\right)_{y}+\lambda\right]$. Because $v_{i+1} v_{i+2} \notin E\left(L_{n}\right)$, we must then have $\left|\left(p\left(v_{i+1}\right)\right)_{x}-\left(p\left(v_{i+2}\right)\right)_{x}\right|>\lambda$. Moreover, since $P\left(u_{i+1}\right)$ contain either the top-right or bottom-right corners of $P\left(v_{i+1}\right)$, we must have $\left(p\left(v_{i+1}\right)\right)_{x} \in\left[\left(p\left(u_{i+1}\right)\right)_{x}-\lambda,\left(p\left(u_{i+1}\right)\right)_{x}\right]$. On the other hand, since $v_{i+2} u_{i+1} \in E\left(L_{n}\right)$ we must have $\left(p\left(v_{i+2}\right)\right)_{x} \in\left[\left(p\left(u_{i+1}\right)\right)_{x}-\lambda,\left(p\left(u_{i+1}\right)\right)_{x}+\lambda\right]$. Because $v_{i+1} v_{i+2} \notin E\left(L_{n}\right)$ we thus have $\left(p\left(v_{i+1}\right)\right)_{x}+\lambda<\left(p\left(v_{i+2}\right)\right)_{x}$, so that all three lines of (4) hold also for $i+1$.

We now combine the observations for the graphs $O_{n}$ and $L_{n}$ into the lowerbound results we are after.

THEOREM 5. If $P$ is a parallelogram with rational corner points, then $t_{P}(n)=$ $\Omega\left(n^{2}\right)$.

Proof. By Corollary 1, it suffices to prove the theorem for the special case when $P$ is the unit square $U$. Let $G$ be the (vertex-) disjoint union of $O_{n}$ and $L_{n}$. (That is, $G$ consists of a copy of $O_{n}$ and a copy of $L_{n}$ on disjoint vertex sets with no edges between them.) Then $G$ is a unit square translate graph and it has $\left|V\left(O_{n}\right)\right|+\left|V\left(L_{n}\right)\right|=$ $(2 n+2)+(3 n-2)=O(n)$ vertices. Suppose that $K$ is such that $G$ can be represented as a $\lambda U$-translate graph for some $\lambda$ with all corner points on a $K \times K$ subgrid of $\mathbb{Z}^{2}$. By Lemmas 5 and 6 , we must then have $K=\Omega\left(n^{2}\right)$.

4. Lower bound for $\boldsymbol{P}$-translate graphs when $\boldsymbol{P}$ is not a parallelogram. In this section, we aim to prove the following result: if $P$ is an arbitrary convex polygon (with rational corner points) that is not a parallelogram, then $t_{P}(n)=2^{\Omega(n)}$.

In section 4.1 we first give some preliminaries from the theory of oriented line arrangements and prove an auxiliary lemma that we need in our construction. In section 4.2 we use the lemma to define a "hard" family of $P$-translate graphs, which will give us the lower bound result for $P$-translate graphs when $P$ is not a parallelogram.

4.1. Preliminaries: Line arrangements and sign vectors. A line $\ell$ in the plane partitions $\mathbb{R}^{2} \backslash \ell$ into two parts. In an oriented line we (arbitrarily) label one of these components $\ell^{+}$, the "positive side," and the other $\ell^{-}$, the "negative side." An oriented line arrangement $\mathcal{L}=\left(\ell_{1}, \ldots, \ell_{n}\right)$ is a collection of oriented lines in the plane. The sign vector $\sigma(p ; \mathcal{L}) \in\{-1,0,1\}^{|\mathcal{L}|}$ of a point $p \in \mathbb{R}^{2}$ with respect to an oriented line arrangement $\mathcal{L}$ is given by

$$
(\sigma(p ; \mathcal{L}))_{i}:=\left\{\begin{array}{cl}
-1 & \text { if } p \in \ell_{i}^{-} \\
0 & \text { if } p \in \ell_{i} \\
+1 & \text { if } p \in \ell_{i}^{+}
\end{array}\right.
$$

If $W \subseteq \mathbb{R}^{2}$ is a set of points, then we write

$$
\sigma(W ; \mathcal{L}):=\{\sigma(p ; \mathcal{L}): p \in W\}
$$

If $\mathcal{L}$ is an oriented line arrangement, then we will call $\mathcal{D}(\mathcal{L}):=\sigma\left(\mathbb{R}^{2}, \mathcal{L}\right)$ the combinatorial description of $\mathcal{L}$. In words, $\mathcal{D}(\mathcal{L})$ is the set of all sign vectors that occur. If $\mathcal{L}, \mathcal{L}^{\prime}$ are two oriented line arrangements with $\mathcal{D}(\mathcal{L})=\mathcal{D}\left(\mathcal{L}^{\prime}\right)$, then we say that they are isomorphic. 


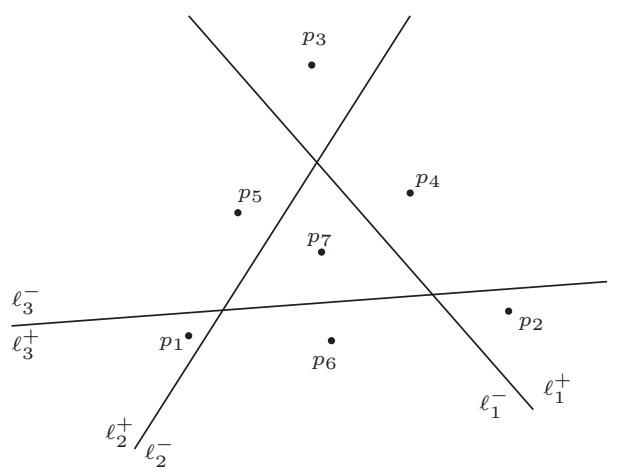

FIG. 3. The position of the oriented lines and points in Lemma 7.

If $\mathcal{L}=\left(\ell_{1}, \ldots, \ell_{n}\right)$ is an oriented line arrangement, then $\mathbb{R}^{2} \backslash\left(\ell_{1} \cup \cdots \cup \ell_{n}\right)$ consists of connected regions, called cells. There is a 1-1 correspondence between cells and sign vectors which do not contain zero (i.e., the set of points in a given cell is precisely the set of points with a specific zero-free sign vector). Similarly, the intersection points between lines correspond to sign vectors with two or more zeroes and each sign vector with exactly one zero corresponds to a line segment (i.e., a connected component of $\ell_{i} \backslash\left(\ell_{1} \cup \cdots \cup \ell_{i-1} \cup \ell_{i+1} \cup \cdots \cup \ell_{n}\right)$ for some $\left.1 \leq i \leq n\right)$. Let us also remark that all relevant combinatorial information, such as whether two cells are incident, whether a segment is bounded, or whether a certain cell is a triangle, can be recovered from the combinatorial description.

The following standard elementary observation will be needed several times in this section.

LEMma 7. Let $\ell_{1}, \ell_{2}, \ell_{3}$ be oriented lines and suppose there exist points $p_{1}, \ldots, p_{7} \in$ $\mathbb{R}^{2}$ such that

$$
\begin{array}{lll}
p_{1} \in \ell_{1}^{-} \cap \ell_{2}^{+} \cap \ell_{3}^{+}, & p_{2} \in \ell_{1}^{+} \cap \ell_{2}^{-} \cap \ell_{3}^{+}, & p_{3} \in \ell_{1}^{+} \cap \ell_{2}^{+} \cap \ell_{3}^{-}, \\
p_{4} \in \ell_{1}^{+} \cap \ell_{2}^{-} \cap \ell_{3}^{-}, & p_{5} \in \ell_{1}^{-} \cap \ell_{2}^{+} \cap \ell_{3}^{-}, & p_{6} \in \ell_{1}^{-} \cap \ell_{2}^{-} \cap \ell_{3}^{+}, \\
p_{7} \in \ell_{1}^{-} \cap \ell_{2}^{-} \cap \ell_{3}^{-} . & &
\end{array}
$$

(See Figure 3 for a depiction.) Then $\ell_{1}^{-} \cap \ell_{2}^{-} \cap \ell_{3}^{-}$is an (open) triangle. Moreover, $\ell_{1}^{-} \cap \ell_{2}^{-} \cap \ell_{3}^{-} \subseteq \operatorname{conv}\left(\left\{p_{1}, p_{2}, p_{3}\right\}\right)$.

Proof. It follows from standard results on oriented line arrangements (for instance, Lemmas 6 and 7 in [12]) that the seven sign vectors of the points $p_{1}, \ldots, p_{7}$ completely determine the combinatorial description of the line arrangement $\mathcal{L}=\left(\ell_{1}, \ell_{2}, \ell_{3}\right)$. Put differently, from these seven sign vectors we can recover all other sign vectors of the line arrangement. In particular, $\mathcal{L}$ must be isomorphic to the line arrangement shown in Figure 3. Hence $\ell_{1}^{-} \cap \ell_{2}^{-} \cap \ell_{3}^{-}$is indeed an (open) triangle. We also have that $\ell_{2}, \ell_{3}$ divide $\ell_{1}$ into the three segments $\ell_{1} \cap \ell_{2}^{+}, \ell_{1} \cap \ell_{2}^{-} \cap \ell_{3}^{-}, \ell_{3} \cap \ell_{3}^{+}$, where $\ell_{1} \cap \ell_{2}^{-} \cap \ell_{3}^{-}$is bounded and the others unbounded.

Let $a, b, c$ be defined by $\{a\}=\ell_{1} \cap \ell_{2},\{b\}=\ell_{1} \cap \ell_{3},\{c\}=\ell_{2} \cap \ell_{3}$. (So they are the corners of the triangle $\ell_{1}, \ell_{2}, \ell_{3}$.) Observe that the line segment $\left[p_{1}, p_{2}\right]$ between $p_{1}$ and $p_{2}$ must cross $\ell_{1}$ in a point of $\ell_{1} \cap \ell_{3}^{+}$(as both $p_{1}, p_{2}$ lie in $\ell_{3}^{+}$and they lie on either side of $\left.\ell_{1}\right)$. Similarly, $\left[p_{1}, p_{3}\right]$ crosses $\ell_{1}$ in a point of $\ell_{1} \cap \ell_{2}^{+}$. It follows that $[b, c] \subseteq \operatorname{conv}\left(\left\{p_{1}, p_{2}, p_{3}\right\}\right)$, since the latter contains points on $\ell_{1}$ on either side of $[b, c]$. Completely analogously we also have $[a, b],[a, c] \subseteq \operatorname{conv}\left(\left\{p_{1}, p_{2}, p_{3}\right\}\right)$. It follows that $\ell_{1}^{-} \cap \ell_{2}^{-} \cap \ell_{3}^{-} \subseteq \operatorname{conv}(\{a, b, c\}) \subseteq \operatorname{conv}\left(\left\{p_{1}, p_{2}, p_{3}\right\}\right)$, as required. 

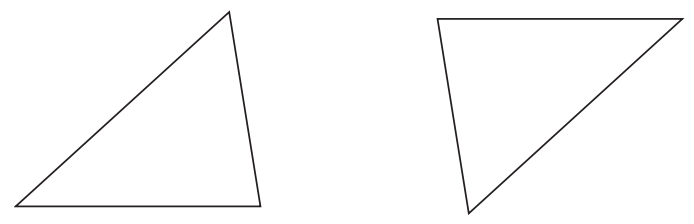

FIG. 4. With three slopes, two kinds of triangles can be formed up to translation and scaling.

The slope of a line or line segment is an angle $s \in\left(-\frac{\pi}{2}, \frac{\pi}{2}\right]$, where $s=0$ means "horizontal" and $s=\frac{\pi}{2}$ means "vertical."

4.1.1. An auxiliary lemma on sign vectors. The following lemma is pivotal for the construction of a family of $P$-translate graphs that need large grids. The lemma shows that if we fix a finite set of slopes, then it is possible to choose a set of sign vectors $\mathcal{S}$ with the following properties. The sign vectors occur as sets $\mathcal{S}=\sigma(W, \mathcal{L})$ for some set of points $W$ and oriented line arrangement $\mathcal{L}$ with the additional property that every line of $\mathcal{L}$ has a slope from our predetermined set of slopes and no point of $W$ lies on a line of $\mathcal{L}$. The sign vectors in $\mathcal{S}$ have the property that, whenever $W \subseteq \mathbb{R}^{2}$ is a nonempty set of points and $\mathcal{L}$ is a line arrangement using only slopes from our predetermined set of slopes and $\mathcal{S}=\sigma(W, \mathcal{L})$, then the point set $W$ "needs a large grid," as there are four distinct points $p, q, r, s$ such that $\|p-q\| /\|r-s\|$ is exponentially large. Moreover, even if we "disregard" any small subset of the points and the lines (which amounts to removing some lines and points from the configuration), then this last property still holds.

Lemma 8. For $k \geq 3$, let $s_{1}, \ldots, s_{k}$ be distinct slopes. There are constants $\alpha=\alpha\left(s_{1}, \ldots, s_{k}\right)>1$ and $m_{0}=m_{0}\left(s_{1}, \ldots, s_{k}\right) \in \mathbb{N}$ such that the following hold. For all $m \geq m_{0}$ there exists an oriented line arrangement $\mathcal{L}=\left(\ell_{1}, \ldots, \ell_{3 m}\right)$ and a set of points $W=\left\{p_{1}, \ldots, p_{7 m}\right\} \subseteq \mathbb{R}^{2}$ with the following properties:

(i) $\sigma(W ; \mathcal{L}) \subseteq\{-1,+1\}^{3 m}$, and

(ii) each line of $\mathcal{L}$ has slope $s_{1}, s_{2}$ or $s_{3}$, and

(iii) for every $A \subseteq\{1, \ldots, 3 m\}, B \subseteq\{1, \ldots, 7 m\}$ with $|A| \geq 2.99 \cdot m,|B| \geq 6.99 \cdot m$ and every oriented line arrangement $\tilde{\mathcal{L}}=\left(\tilde{\ell}_{1}, \ldots, \tilde{\ell}_{3 m}\right)$ and point set $\tilde{W}=$ $\left\{\tilde{p}_{1}, \ldots, \tilde{p}_{7 m}\right\} \subseteq \mathbb{R}^{2}$ such that

(a) $\sigma\left(\tilde{p}_{i} ; \tilde{\ell}_{j}\right)=\sigma\left(p_{i} ; \ell_{j}\right)$ for all $i \in B, j \in A$, and

(b) Each line of $\tilde{\mathcal{L}}$ has slope $s_{1}, \ldots, s_{k-1}$ or $s_{k}$,

there exist distinct points $p, q, r, s \in \tilde{W}$ such that $\|p-q\| /\|r-s\|>\alpha^{m}$.

Proof. Let $T$ be an open triangle whose sides have slopes $s_{1}, s_{2}, s_{3}$. Observe that with slopes $s_{1}, s_{2}, s_{3}$ two different kinds of (open) triangles can be formed, those homothetic to $T$ and those homothetic to $-T$, the reflection of $T$ through the origin. See Figure 4 for a depiction.

We start with a sequence of (open) triangles $T_{1} \supseteq T_{2} \supseteq \cdots \supseteq T_{m}$, where $T_{i}$ is a homothet of $T$ when $i$ is odd and a homothet of $-T$ if $i$ is even. We also require that $\operatorname{cl}\left(T_{i+1}\right) \subseteq T_{i}$ for all $i=1, \ldots, m-1$.

We now place oriented lines $\ell_{1}, \ldots, \ell_{3 m}$ in such a way that

$$
T_{i}=\ell_{3 i-2}^{-} \cap \ell_{3 i-1}^{-} \cap \ell_{3 i}^{-},
$$

and $\ell_{3 i-2}$ has slope $s_{1}, \ell_{3 i-1}$ has slope $s_{2}$ and $\ell_{3 i}$ has slope $s_{3}$. Let us also place points 


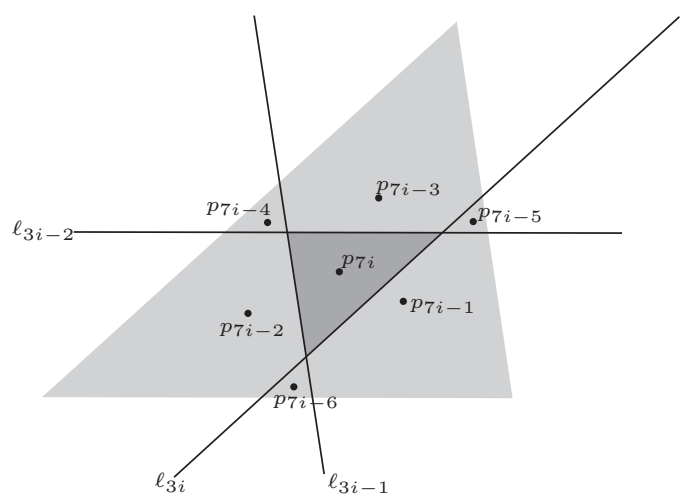

FIG. 5. The placement of $\ell_{3 i-2}, \ell_{3 i-1}, \ell_{3 i}$, and $p_{7 i-6}, \ldots, p_{7 i}$. The light triangle represents $T_{i-1}$ and the dark triangle represents $T_{i}$.

$p_{1}, \ldots, p_{7 m}$ such that

$$
\begin{array}{ll}
p_{7 i-6} \in T_{i-1} \cap \ell_{3 i-2}^{-} \cap \ell_{3 i+1}^{+} \cap \ell_{3 i}^{+}, & p_{7 i-5} \in T_{i-1} \cap \ell_{3 i-2}^{+} \cap \ell_{3 i-1}^{-} \cap \ell_{3 i}^{+}, \\
p_{7 i-4} \in T_{i-1} \cap \ell_{3 i-2}^{+} \cap \ell_{3 i-1}^{+} \cap \ell_{3 i}^{-}, & p_{7 i-3} \in T_{i-1} \cap \ell_{3 i-2}^{+} \cap \ell_{3 i-1}^{-} \cap \ell_{3 i}^{-}, \\
p_{7 i-2} \in T_{i-1} \cap \ell_{3 i-2}^{-} \cap \ell_{3 i-1}^{+} \cap \ell_{3 i}^{-}, & p_{7 i-1} \in T_{i-1} \cap \ell_{3 i-2}^{-} \cap \ell_{3 i-1}^{-} \cap \ell_{3 i}^{+}, \\
p_{7 i} \in T_{i-1} \cap \ell_{3 i-2}^{-} \cap \ell_{3 i-1}^{-} \cap \ell_{3 i}^{-}, &
\end{array}
$$

where we set $T_{0}:=\mathbb{R}^{2}$. Note that the points $p_{7 i-6}, \ldots, p_{7 i}$ can be placed in this way, because every cell of the oriented line arrangement $\left(\ell_{3 i-2}, \ell_{3 i-1}, \ell_{3 i}\right)$ intersects $T_{i-1}$ (recall $\left.\mathrm{cl}\left(T_{i}\right) \subseteq T_{i-1}\right)$. See Figure 5 for a depiction of the construction.

Let us also point out that, as $\ell_{3 i-2}$ and $\ell_{3 i+1}$ are parallel and $T_{i+1} \subseteq T_{i}$ is a homothet of $-T_{i}$, in the construction we have

$$
p_{7 i-6} \in \ell_{3 i+1}^{+}, \quad p_{7 i-5}, p_{7 i-4} \in \ell_{3 i+1}^{-} .
$$

Similar statements can be made about the position of $p_{7 i-6}, p_{7 i-5}, p_{7 i-4}$ with respect to $\ell_{3 i+2}$ and $\ell_{3 i+3}$.

Set $W:=\left\{p_{1}, \ldots, p_{7 m}\right\}$ and $\mathcal{L}:=\left(\ell_{1}, \ldots, \ell_{3 m}\right)$. This gives us the sets $W, \mathcal{L}$. Observe that parts (i) and (ii) of the lemma hold by construction.

Now let $A \subseteq\{1, \ldots, 3 m\}, B \subseteq\{1, \ldots, 7 m\}, \tilde{W}=\left\{\tilde{p}_{1}, \ldots, \tilde{p}_{7 m}\right\} \subseteq \mathbb{R}^{2}$, and $\tilde{\mathcal{L}}=$ $\left(\tilde{\ell}_{1}, \ldots, \tilde{\ell}_{3 m}\right)$ be as in part (iii) of the lemma. Let us set

$$
I:=\{i \in \mathbb{N}: i \text { odd, and }\{3 i-2, \ldots, 3 i+3\} \subseteq A \text {, and }\{7 i-6, \ldots, 7 i+7\} \subseteq B\},
$$

and let $J:=\{i, i+1: i \in I\}$. Relabel $J$ as $j_{1}<j_{2}<\cdots<j_{\tilde{m}}$. Note that the following properties hold:

(i) $3 j_{i}-2,3 j_{i}-1,3 j_{i} \in A$ for all $i=1, \ldots, \tilde{m}$;

(ii) $7 j_{i}-6, \ldots, 7 j_{i} \in B$ for all $i=1, \ldots, \tilde{m}$;

(iii) $j_{i}$ is even if $i$ is even and odd if $i$ is odd;

(iv) $\tilde{m}>0.96 m$.

(The first three properties follow directly from the definition of $J$ and the last follows because $|A|>2.99 \mathrm{~m},|B|>6.99 \mathrm{~m}$ so that at most $0.02 \mathrm{~m}$ odd integers between one and $m$ are missing from $I$ - so that at most $0.04 m$ integers between one and $m$ are missing from $J$.)

Let us set

$$
A^{\prime}:=\{3 j-2,3 j-1,3 j: j \in J\}, \quad B^{\prime}:=\{7 j-6, \ldots, 7 j: j \in J\},
$$


and

$$
W^{\prime}:=\left\{\tilde{p}_{i}: i \in B^{\prime}\right\}, \quad \mathcal{L}^{\prime}:=\left(\tilde{\ell}_{i}\right)_{i \in A^{\prime}} .
$$

Observe that $T_{j_{i}}$ is a homothet of $T$ for odd $i$ and a homothet of $-T$ for even $i$. Therefore, up to a relabeling, $\mathcal{S}^{\prime}:=\sigma\left(W^{\prime} ; \mathcal{L}^{\prime}\right)$ is exactly the set of sign vectors $\mathcal{S}$ we would have obtained from our construction above if we had started the construction with $T_{j_{1}} \supseteq T_{j_{2}} \supseteq \cdots \supseteq T_{j_{\tilde{m}}}$ instead of $T_{1} \supseteq \cdots \supseteq T_{m}$. Similarly, again up to a relabeling, $\mathcal{S}^{\prime}$ is exactly the set of sign vectors we would have ended up with if we had started with $T_{1} \supseteq \cdots \supseteq T_{\tilde{m}}$.

For the remainder of the proof let us discard all points not in $W^{\prime}$ and all lines not in $\mathcal{L}^{\prime}$ and relabel the remaining points and lines in such a way that $j_{i}=i$. In other words, we have a point set $\tilde{W}=\left\{\tilde{p}_{1}, \ldots, \tilde{p}_{7 \tilde{m}}\right\}$ and a line arrangement $\tilde{\mathcal{L}}=\left(\tilde{\ell}_{1}, \ldots, \tilde{\ell}_{3} \tilde{m}\right)$ such that $\sigma(\tilde{W}, \tilde{\mathcal{L}})$ equals (up to relabeling) $\mathcal{S}^{\prime}$. For $i=1, \ldots, \tilde{m}$ let us set

$$
\tilde{T}_{i}:=\tilde{\ell}_{3 i-2}^{-} \cap \tilde{\ell}_{3 i-1}^{-} \cap \tilde{\ell}_{3 i}^{-} .
$$

It follows from the choice of the original lines and points (i.e., (5)) and Lemma 7 that $\tilde{T}_{i}$ is a triangle and that

$$
\tilde{T}_{i} \subseteq \operatorname{conv}\left(\left\{\tilde{p}_{7 i-6}, \tilde{p}_{7 i-5}, \tilde{p}_{7 i-4}\right\}\right) \text { for all } 1 \leq i \leq \tilde{m} .
$$

Also, note that since each line of $\tilde{\mathcal{L}}$ has a slope in $s_{1}, \ldots, s_{k}$, the sides of $\tilde{T}_{i}$ also have slopes $s_{1}, \ldots, s_{k}$. From the sign vectors of $\tilde{p}_{7 i-6}, \tilde{p}_{7 i-5}, \tilde{p}_{7 i-4}$ it follows that they lie in $\tilde{T}_{i-1}$ for all $1<i \leq \tilde{m}$. Together with $(7)$ this gives that

$$
\operatorname{cl}\left(\tilde{T}_{i}\right) \subseteq \tilde{T}_{i-1} \quad \text { for all } 1<i \leq \tilde{m} .
$$

We also need the following observation.

CLAIM. $\tilde{T}_{i+1}$ is not a homothet of $\tilde{T}_{i}$.

Proof of claim. Let the slopes of $\tilde{\ell}_{3 i-2}, \tilde{\ell}_{3 i-1}, \tilde{\ell}_{3 i}$ be $s_{a}, s_{b}, s_{c}$, and let the slopes of $\tilde{\ell}_{3 i+1}, \tilde{\ell}_{3 i+2}, \tilde{\ell}_{3 i+3}$ be $s_{u}, s_{v}, s_{w}$.

If $\left\{s_{a}, s_{b}, s_{c}\right\} \neq\left\{s_{u}, s_{v}, s_{w}\right\}$, then we are clearly done, so let us assume that $\left\{s_{a}, s_{b}, s_{c}\right\}=\left\{s_{u}, s_{v}, s_{w}\right\}$. From the fact that $\sigma(\tilde{W}, \tilde{\mathcal{L}})$ agrees with the sign vectors of the original construction and (5) and (6), we have

$$
\begin{aligned}
& \sigma\left(\tilde{p}_{7 i-6} ;\left(\tilde{\ell}_{3 i-2}, \tilde{\ell}_{3 i-1}, \tilde{\ell}_{3 i}, \tilde{\ell}_{3 i+1}\right)\right)=(-1,+1,+1,+1), \\
& \sigma\left(\tilde{p}_{7 i-5} ;\left(\tilde{\ell}_{3 i-2}, \tilde{\ell}_{3 i-1}, \tilde{\ell}_{3 i}, \tilde{\ell}_{3 i+1}\right)\right)=(+1,-1,+1,-1), \\
& \sigma\left(\tilde{p}_{7 i-4} ;\left(\tilde{\ell}_{3 i-2}, \tilde{\ell}_{3 i-1}, \tilde{\ell}_{3 i}, \tilde{\ell}_{3 i+1}\right)\right)=(+1,+1,-1,-1) .
\end{aligned}
$$

Similarly we also have that $\tilde{p}_{7 i+1} \in \tilde{T}_{i} \cap \tilde{\ell}_{3 i+1}^{-}$and $\tilde{p}_{7 i+2} \in \tilde{T}_{i} \cap \tilde{\ell}_{3 i+1}^{+}$. Thus $\tilde{\ell}_{3 i+1}$ strictly separates two points in $\tilde{T}_{i}$, which implies

$$
\tilde{\ell}_{3 i+1} \text { intersects } \tilde{T}_{i} \text {. }
$$

For convenience, let us assume the slope of $\tilde{\ell}_{3 i-2}$ corresponds to "horizontal" and that $\tilde{\ell}_{3 i-2}^{-}$lies "above" $\tilde{\ell}_{3 i-2}$. (Hence $\tilde{p}_{7 i-6}$ and $\tilde{T}_{i}$ are also above $\tilde{\ell}_{3 i-2}$.)

First suppose that $\tilde{\ell}_{3 i+1}$ is parallel to $\tilde{\ell}_{3 i-1}$. From (10) and the fact that $\tilde{p}_{7 i-6}, \tilde{p}_{7 i-4}$ $\in \tilde{\ell}_{3 i-1}^{+}$by (9), it then follows that $\tilde{p}_{7 i-6}, \tilde{p}_{7 i-4}$ lie on the same side of $\tilde{\ell}_{3 i+1}$. (See Figure 6 for a depiction).

But this contradicts that $\tilde{p}_{7 i-6} \in \tilde{\ell}_{3 i+1}^{+}, \tilde{p}_{7 i-4} \in \tilde{\ell}_{3 i+1}^{-}$, also by (9). Hence we must have that $\tilde{\ell}_{3 i+1}$ is not parallel to $\tilde{\ell}_{3 i-1}$. Similarly, $\tilde{\ell}_{3 i+1}$ cannot be parallel to $\tilde{\ell}_{3 i}$. 


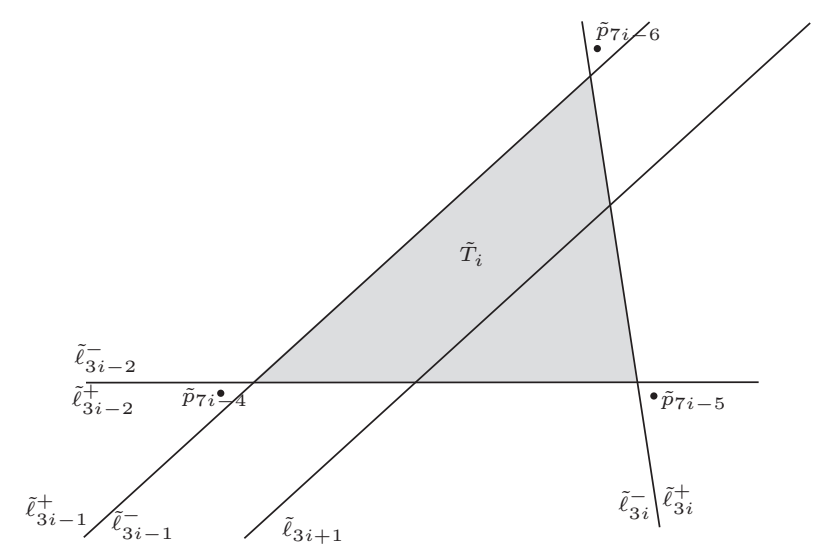

FIG. 6. The triangle $\tilde{T}_{i}$ and the line $\tilde{\ell}_{3 i+1}$ when $\tilde{\ell}_{3 i-1}$ and $\tilde{\ell}_{3 i+1}$ are parallel.

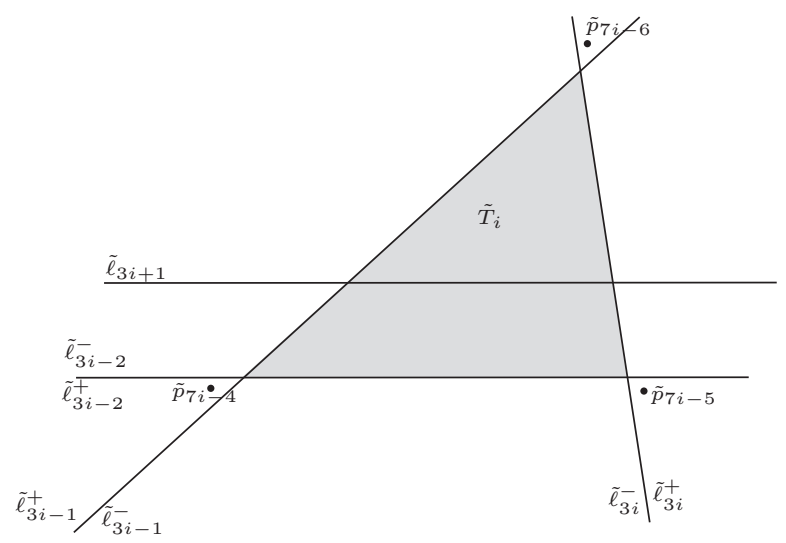

FIG. 7. The triangle $\tilde{T}_{i}$ and the line $\tilde{\ell}_{3 i+1}$ when $\tilde{\ell}_{3 i+1}$ is parallel to $\tilde{\ell}_{3 i-2}$.

It follows that $\tilde{\ell}_{3 i-2}, \tilde{\ell}_{3 i+1}$ are both horizontal. The points $\tilde{p}_{7 i-5}, \tilde{p}_{7 i-4}$ lie in $\tilde{\ell}_{3 i-2}^{+}$, which is below $\tilde{\ell}_{3 i-2}$. On the other hand we also have that $\tilde{p}_{7 i-5}, \tilde{p}_{7 i-4} \in \tilde{\ell}_{3 i+1}^{-}$and $\tilde{\ell}_{3 i+1}$ is contained in $\tilde{\ell}_{3 i-2}^{-}$(it is parallel to $\tilde{\ell}_{3 i-2}$ and intersects $T_{i}$ ). This gives that $\tilde{\ell}_{3 i+1}^{-}$is below $\tilde{\ell}_{3 i+1}$. (See Figure 7 for a depiction.)

Hence $\tilde{T}_{i+1}=\tilde{\ell}_{3 i+1}^{-} \cap \tilde{\ell}_{3 i+2}^{-} \cap \tilde{\ell}_{3 i+3}^{-}$lies below $\tilde{\ell}_{3 i+1}$. But then $\tilde{T}_{i}, \tilde{T}_{i+1}$ cannot be homothets, since in $T_{i}$ the horizontal segment is the lowest part of the triangle and in $T_{i+1}$ the horizontal segment is the highest part of the triangle).

Now let $D_{1}, \ldots, D_{K}$ with $K=2\left(\begin{array}{c}k \\ 3\end{array}\right)$ be all possible triangles, up to translations and scalings, that have slopes $\in\left\{s_{1}, \ldots, s_{k}\right\}$. Let us set

$$
\beta:=\max _{i \neq j} \max _{\substack{D^{\prime} \subseteq D_{j}, D^{\prime} \in \mathcal{H}\left(D_{i}\right)}} \frac{\operatorname{area}\left(D^{\prime}\right)}{\operatorname{area}\left(D_{j}\right)},
$$

where $\mathcal{H}(A):=\left\{x+\lambda A: x \in \mathbb{R}^{2}, \lambda>0\right\}$ denotes the set of all $A$-homothets. Clearly $\beta<1$ and $\beta$ depends only on $s_{1}, \ldots, s_{k}$. By the claim and the observations that precede it, we now have

$$
\operatorname{area}\left(\tilde{T}_{i+1}\right) \leq \beta \cdot \operatorname{area}\left(\tilde{T}_{i}\right) \quad \text { for all } i=1, \ldots, \tilde{m}-1 .
$$


There must be a set $J^{\prime} \subseteq J$ with $\left|J^{\prime}\right| \geq \tilde{m} / K$ such that $\tilde{T}_{i}, \tilde{T}_{j}$ are homothets for all $i, j \in J^{\prime}$. So in particular there are indices $1 \leq i_{1}, i_{2} \leq \tilde{m}$ such that $i_{2} \geq i_{1}+\tilde{m} / K-1$ and $T_{i_{1}}, T_{i_{2}}$ are homothets. Then we have that

$$
\operatorname{area}\left(\tilde{T}_{i_{2}}\right) \leq \beta^{\tilde{m} / K-1} \cdot \operatorname{area}\left(\tilde{T}_{i_{1}}\right) .
$$

Since $\tilde{T}_{i_{1}}, \tilde{T}_{i_{2}}$ are homothets we must have

$$
\left(\frac{\operatorname{diam}\left(\tilde{T}_{i_{1}}\right)}{\operatorname{diam}\left(\tilde{T}_{i_{2}}\right)}\right)^{2}=\frac{\operatorname{area}\left(\tilde{T}_{i_{1}}\right)}{\operatorname{area}\left(\tilde{T}_{i_{2}}\right)} .
$$

Together with (11) this gives

$$
\operatorname{diam}\left(\tilde{T}_{i_{2}}\right) \leq \beta^{\frac{1}{2}(\tilde{m} / K-1)} \cdot \operatorname{diam}\left(\tilde{T}_{i_{1}}\right)
$$

Since $\tilde{T}_{i_{1}} \subseteq \operatorname{conv}\left(\left\{\tilde{p}_{7 i_{1}-6}, \tilde{p}_{7 i_{1}-5}, \tilde{p}_{7 i_{1}-4}\right\}\right)$ by (7), we can assume (up to relabeling) that $\left\|\tilde{p}_{7 i_{1}-5}-\tilde{p}_{7 i_{1}-4}\right\| \geq \operatorname{diam}\left(\tilde{T}_{i_{1}}\right)$. Since $\tilde{p}_{7 i_{2}+1}, \tilde{p}_{7 i_{2}+2} \in \tilde{T}_{i_{2}}$ are distinct (they have distinct sign vectors with respect to $\tilde{\mathcal{L}})$, we have that $\left\|\tilde{p}_{7 i_{2}+1}-\tilde{p}_{7 i_{2}+2}\right\|>0$. Setting $\alpha:=\beta^{-\frac{1}{100 K}}$, we see that

$$
\left\|\tilde{p}_{7 i_{1}-6}-\tilde{p}_{7 i_{1}-5}\right\| /\left\|\tilde{p}_{7 i_{2}+1}-\tilde{p}_{7 i_{2}+2}\right\| \geq \beta^{-\frac{1}{2}(\tilde{m} / K-1)} \geq \alpha^{m}
$$

for $m$ sufficiently large (for $m /(100 K) \leq 0.96 \cdot m /(2 K)-\frac{1}{2}$ to hold). This concludes the proof of the lemma.

4.2. A family of $\boldsymbol{P}$-translate graphs. Recall that we aim at a lower bound for $t_{P}(n)$ in the case when $P$ is a convex polygon other than a parallelogram with rational corner points. We are now ready to describe the construction of a $P$-translate graph that will need a large integer grid size. We first give the construction and then prove an exponential lower bound on the grid size of any realization of the graphs.

4.2.1. Defining the $\boldsymbol{P}$-translate graphs. Let $P$ be a convex polygon that is not a parallelogram. Define $B$ as the Minkowski sum of $P$ and $-P$ :

$$
B:=P+(-P) \text {. }
$$

Observe that

$$
\left(P+z_{1}\right) \cap\left(P+z_{2}\right) \neq \emptyset \text { if and only if } z_{1} \in z_{2}+B .
$$

(To see this observe that $z_{1} \in z_{2}+B$ if and only if $z_{1}-z_{2} \in B$ if and only if there exist $y_{1}, y_{2} \in P$ such that $z_{1}-z_{2}=y_{2}-y_{1}$ if and only if there exist $y_{1}, y_{2} \in P$ such that $y_{1}+z_{1}=y_{2}+z_{2}$ if and only if $\left(z_{1}+P\right) \cap\left(z_{2}+P\right) \neq \emptyset$.) Let us point out that the position of $P$ with respect to the origin is irrelevant for the definition of $B$ since if $P^{\prime}$ is any translate of $P$, then $P^{\prime}+\left(-P^{\prime}\right)=P+(-P)$. Observe that $B$ is centrally symmetric with respect to the origin and that $B$ will have two sides for each slope that occurs as the slope of some side of $P$. (See Figure 8 for a depiction.)

Let $W=\left\{p_{1}, \ldots, p_{7 m}\right\}, \mathcal{L}=\left(\ell_{1}, \ldots, \ell_{3 m}\right)$ be as provided by Lemma 8 , where $s_{1}, \ldots, s_{k}$ are the slopes occurring on the sides of $P$ (and $B$ ).

Now let $\varepsilon_{1}, \varepsilon_{2}>0$ be small constants with $\varepsilon_{1} \ll \varepsilon_{2}$, to be chosen later, and let $N \in \mathbb{N}$ be a large constant. Note $\varepsilon_{1}, \varepsilon_{2}, N$ depend only on $P$ and not on the number of lines $m$. We can assume without loss of generality that $\operatorname{diam}(W)<\varepsilon_{1}$.

Copyright (C) by SIAM. Unauthorized reproduction of this article is prohibited. 

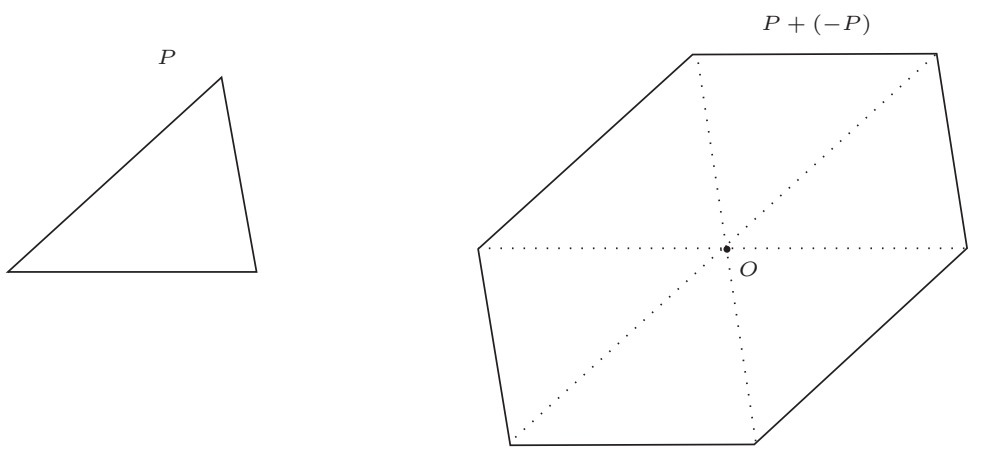

Fig. 8. A polygon $P$ and the corresponding $B=P+(-P)$.

Let us pick vectors $v_{1}, v_{2}, v_{3}$ of length $\left\|v_{1}\right\|=\left\|v_{2}\right\|=\left\|v_{3}\right\|=\varepsilon_{2}$, where the slope of $v_{i}$ is $s_{i}$. For $x=\left(x_{1}, x_{2}, x_{3}\right) \in\{0, \ldots, N\}^{3}$ let us set

$$
\begin{aligned}
& p_{i}(x):=p_{i}+x_{1} v_{1}+x_{2} v_{2}+x_{3} v_{3} \\
& \ell_{i}(x):=\ell_{i}+x_{1} v_{1}+x_{2} v_{2}+x_{3} v_{3} .
\end{aligned}
$$

That is, we translate the point, resp., oriented line, by the vector $x_{1} v_{1}+x_{2} v_{2}+x_{3} v_{3}$. Here we "keep the orientation" in the obvious way, i.e., in the sense that $\ell_{i}^{+}(x)=$ $\ell_{i}^{+}+x_{1} v_{1}+x_{2} v_{2}+x_{3} v_{3}$. Let us also write

$$
\begin{aligned}
W(x) & :=\left\{p_{1}(x), \ldots, p_{7 m}(x)\right\} \\
\mathcal{L}(x) & :=\left(\ell_{1}(x), \ldots, \ell_{3 m}(x)\right) .
\end{aligned}
$$

Clearly

$$
\sigma(W(x) ; \mathcal{L}(x))=\sigma(W ; \mathcal{L})
$$

for all $x$. Also observe that the same oriented line occurs in several $\mathcal{L}(x)$, because if $\ell_{i}$ has slope $s_{1}$, then

$$
\ell_{i}\left(0, x_{2}, x_{3}\right)=\ell_{i}\left(1, x_{2}, x_{3}\right)=\cdots=\ell_{i}\left(N, x_{2}, x_{3}\right)
$$

for all $x_{2}, x_{3}$. Similar statements hold when $\ell_{i}$ has slope $s_{2}$ or $s_{3}$.

For each $i=1, \ldots, 3 m$ and each $x \in\{0, \ldots, N\}^{3}$, we now place points $c_{i}^{-}(x), c_{i}^{+}(x)$ such that, writing $B_{i}^{-}(x):=B+c_{i}^{-}(x), B_{i}^{+}(x):=B+c_{i}^{+}(x)$, we have for every index $1 \leq i \leq 3 m$ and every $x \in\{0, \ldots, N\}^{3}$

(c-1) $B_{i}^{-}(x) \cap B_{i}^{+}(x)=\emptyset$;

(c-2) $\ell_{i}^{+}(x) \cap\left(\bigcup_{y} W(y)\right)=B_{i}^{+}(x) \cap\left(\bigcup_{y} W(y)\right)$;

(c-3) $\ell_{i}^{-}(x) \cap\left(\bigcup_{y} W(y)\right)=B_{i}^{-}(x) \cap\left(\bigcup_{y} W(y)\right)$;

(c-4) if $\ell_{i}(x)=\ell_{i}(y)$, then $B_{i}^{+}(x)=B_{i}^{+}(y)$ and $B_{i}^{-}(x)=B_{i}^{-}(y)$.

To see that it is possible to place $B_{i}^{-}(x), B_{i}^{+}(x)$ in such a way that these demands are met, observe that provided we chose $\varepsilon_{1}, \varepsilon_{2}$ sufficiently small, the set $\bigcup_{y} W(y)$ has a diameter that is much smaller than the length of any side of $B$. The points of $A^{+}:=\ell_{i}^{+}(x) \cap\left(\bigcup_{y} W(y)\right)$ lie in the open halfspace $\ell_{i}^{+}(x)$, and each of them is thus a positive distance away from $\ell_{i}(x)$. We can now place $B_{i}^{+}(x)$, a translate of $B$, inside $\ell_{i}^{+}(x)$ in such a way that one of the two sides of $B_{i}^{+}$that has slope $s_{1}$ is 


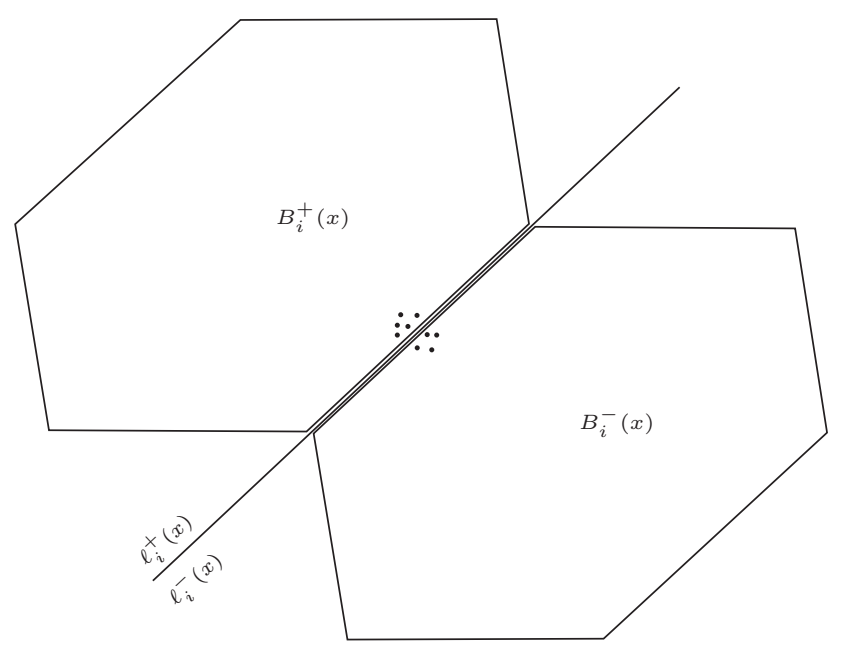

FIG. 9. Placing $B_{i}^{+}(x)$ and $B_{i}^{-}(x)$. The group of points in the middle represents $\bigcup_{y} W(y)$.

very close to $\ell_{i}(x)$ and $B_{i}^{+}(x)$ contains $A^{+}$. (See Figure 9 for a depiction.) We let $c_{i}^{+}(x)$ be the vector over which we have to translate $B$ to get $B_{i}^{+}$. Similarly we define $B_{i}^{-}(x) \subseteq \ell_{i}^{+}(x)$ and $c_{i}^{-}(x)$. Part (c-1) holds by construction since we chose $B_{i}^{-}(x) \subseteq \ell_{i}^{-}(x), B_{i}^{+}(x) \subseteq \ell_{i}^{+}(x)$. To see that we can ensure that part (c-4) also holds, note that if $\ell_{i}(x)=\ell_{i}(y)$ and we have already defined $B_{i}^{+}(x), B_{i}^{-}(x)$, then setting $B_{i}^{+}(y):=B_{i}^{+}(x), B_{i}^{-}(y):=B_{i}^{-}(x)$ works (i.e., $B_{i}^{+}(y), B_{i}^{-}(y)$ satisfy (c-1)-(c-4)).

Provided we chose $\varepsilon_{2}$ sufficiently larger than $\varepsilon_{1}$, we can assume that $\operatorname{conv}(W(x)) \cap$ $\operatorname{conv}(W(y))=\emptyset$ for all $x, y \in\{0, \ldots, N\}^{3}$ with $d_{\mathrm{H}}(x, y)=1$. (Recall that $d_{\mathrm{H}}(.,$. denotes the Hamming distance.)

For each pair $x=\left(x_{1}, x_{2}, x_{3}\right), y=\left(y_{1}, y_{2}, y_{3}\right)$ of Hamming distance one, let us place points $a(x, y), b(x, y)$ in such a way that $B+a(x, y)$ and $B+b(x, y)$ are disjoint, and $W(x) \subseteq B+a(x, y)$ and $W(y) \subseteq B+b(x, y)$. (Provided $\varepsilon_{2}$ was chosen sufficiently larger than $\varepsilon_{1}$, this can be done.) Now define

$$
\begin{aligned}
\mathcal{V}:= & \left\{p_{i}(x): i=1, \ldots, 7 m, x \in\{0, \ldots, N\}^{3}\right\} \\
& \cup\left\{c_{i}^{+}(x), c_{i}^{-}(x): i=1, \ldots, 3 m, x \in\{0, \ldots, N\}^{3}\right\} \\
& \cup\left\{a(x, y), b(x, y): x, y \in\{0, \ldots, N\}^{3} \text { and } d_{\mathrm{H}}(x, y)=1\right\} .
\end{aligned}
$$

Let $\mathcal{A}$ define the corresponding family of $P$-translates,

$$
\mathcal{A}:=\{p+P: p \in \mathcal{V}\}
$$

and let $G_{m, N}$ denote the intersection graph defined by $\mathcal{A}$. Observe that $G_{m, N}$ has $O\left(m N^{3}\right)$ vertices.

4.2.2. The lower bound argument. We now prove that the $P$-intersection graphs $G_{m, N}$ we just defined need exponential-size grids.

THEOREM 6. Let $P$ be an arbitrary polygon with rational corner points that is not a parallelogram. Then $t_{P}(n)=2^{\Omega(n)}$.

Proof. Set $N:=10^{10} \cdot k$, and let $m \in \mathbb{N}$ be arbitrary. Let $G_{m, N}$ be as constructed above, and suppose that for some $\lambda \geq 0$ there is a realization $\tilde{\mathcal{A}}:=\{p+\lambda P: p \in \tilde{\mathcal{V}}\}$ of $G_{m, N}$, where each corner point of each translate $p+\lambda P$ lies on $\mathbb{Z}^{2}$. 
For convenience let us write $\tilde{P}:=\lambda P$ and $\tilde{B}:=\lambda B$. By definition, there is a bijection $p \mapsto \tilde{p}$ from $\mathcal{V}$ to $\tilde{\mathcal{V}}$ such that $(p+P) \cap(q+P) \neq \emptyset$ if and only if $(\tilde{p}+\tilde{P}) \cap(\tilde{q}+\tilde{P}) \neq \emptyset$. (Here $\mathcal{V}$ is as defined by (13).) In other words,

$$
p \in q+B \text { if and only if } \tilde{p} \in \tilde{q}+\tilde{B} .
$$

For $x \in\{0, \ldots, N\}^{3}$ and $1 \leq i \leq 7 m$ let $\tilde{p}_{i}(x)$ denote the image of $p_{i}(x)$ under our bijection, for $x \in\{0, \ldots, N\}^{3}$ and $1 \leq i \leq 7 m$ let $\tilde{c}_{i}^{+}(x), \tilde{c}_{i}^{-}(x)$ denote the images of $c_{i}^{+}(x), c_{i}^{-}(x)$, and for $x, y \in\{0, \ldots, N\}^{3}$ with Hamming distance one let $\tilde{a}(x, y), \tilde{b}(x, y)$ denote the images of $a(x, y), b(x, y)$. We can thus write

$$
\begin{aligned}
\tilde{\mathcal{V}}= & \left\{\tilde{p}_{i}(x): i=1, \ldots, 7 m, x \in\{0, \ldots, N\}^{3}\right\} \\
& \cup\left\{\tilde{c}_{i}^{+}(x), \tilde{c}_{i}^{-}(x): i=1, \ldots, 3 m, x \in\{0, \ldots, N\}^{3}\right\} \\
& \cup\left\{\tilde{a}(x, y), \tilde{b}(x, y): x, y \in\{0, \ldots, N\}^{3} \text { and } d_{\mathrm{H}}(x, y)=1\right\} .
\end{aligned}
$$

Let us also set $\tilde{W}(x):=\left\{\tilde{p}_{i}(x): i=1, \ldots, 7 m\right\}$ so that in particular $\tilde{W}(x)$ is the image of $W(x)$ under our bijection.

Recall that we say that a line $\ell$ strictly separates two sets $A, B$ if they are in different connected components of $\mathbb{R}^{2} \backslash \ell$. In other words, $\ell$ can be oriented in such a way that $A \subseteq \ell^{-}, B \subseteq \ell^{+}$. We have the following key observation.

Claim D-1. Suppose $p, q \in \mathcal{V}$ are such that $p+B$ and $q+B$ are disjoint. Set

$$
\begin{array}{ll}
A_{p}:=\mathcal{V} \cap(p+B), & \tilde{A}_{p}:=\left\{\tilde{a}: a \in A_{p}\right\}, \\
A_{q}:=\mathcal{V} \cap(q+B), & \tilde{A}_{q}:=\left\{\tilde{a}: a \in A_{q}\right\} .
\end{array}
$$

Then there exists a line $\ell$ that strictly separates $\tilde{A}_{p}$ and $\tilde{A}_{q}$.

Proof of D-1. Let us set $\tilde{B}_{p}:=\tilde{p}+\tilde{B}, \tilde{B}_{q}:=\tilde{q}+\tilde{B}$. By (14), we must thus have that

$$
\tilde{A}_{p} \subseteq \tilde{B}_{p} \backslash \tilde{B}_{q}, \quad \tilde{A}_{q} \subseteq \tilde{B}_{q} \backslash \tilde{B}_{p} .
$$

For the moment, let us assume that $\tilde{p}, \tilde{q}$ are such that the collection of all corner points of $\tilde{B}_{p}$ and $\tilde{B}_{q}$ is in general position - i.e., no three of the corner points are collinear. In this case, if $s$ is a side of $\tilde{B}_{p}$ and $s^{\prime}$ is a side of $\tilde{B}_{q}$, then either they intersect in a single point that is not a corner point or they do not intersect at all. Recall that we say that two simple closed curves $\gamma_{1}, \gamma_{2}$ cross in a point $a$ if they meet in $a$ and $\gamma_{1}$ passes from one side of $\gamma_{2}$ to the other in $a$.

Since the boundaries $\partial \tilde{B}_{p}, \partial \tilde{B}_{q}$ are simple closed curves, they cross an even number of times. (This follows from the Jordan curve theorem.) Moreover, since $\tilde{B}_{p}, \tilde{B}_{q}$ are both translates of the same convex set, their boundaries cross at most twice $[1$, Lemma 2.3].

If the number of crossings is zero, then it must be the case that either $\tilde{B}_{p} \varsubsetneqq \tilde{B}_{q}$ or $\tilde{B}_{q} \varsubsetneqq \tilde{B}_{p}$ or $\tilde{B}_{p} \cap \tilde{B}_{q}=\emptyset$. The first two options are clearly impossible since $\tilde{B}_{p}$ is a translate of $\tilde{B}_{q}$. If $\tilde{B}_{p} \cap \tilde{B}_{q}=\emptyset$, then we can apply the hyperplane separation theorem [18, Theorem 1.2.4] to find the required line $\ell$.

Let us thus assume the boundaries cross exactly twice and let $a, b$ be the two points where they cross. Observe that, by our assumption, every point of $\partial \tilde{B}_{p} \cap \partial \tilde{B}_{q}$ is a crossing point. So $a, b$ are the only points of $\partial \tilde{B}_{p} \cap \partial \tilde{B}_{q}$. This gives that $\partial \tilde{B}_{p} \backslash\{a, b\}$ consists of two open $\operatorname{arcs} S_{1}, S_{2}$, and one of these, say, $S_{1}$, is contained in $\tilde{B}_{q}$, while the other is contained in $\mathbb{R}^{2} \backslash \tilde{B}_{q}$. (See Figure 10 for a depiction.) 


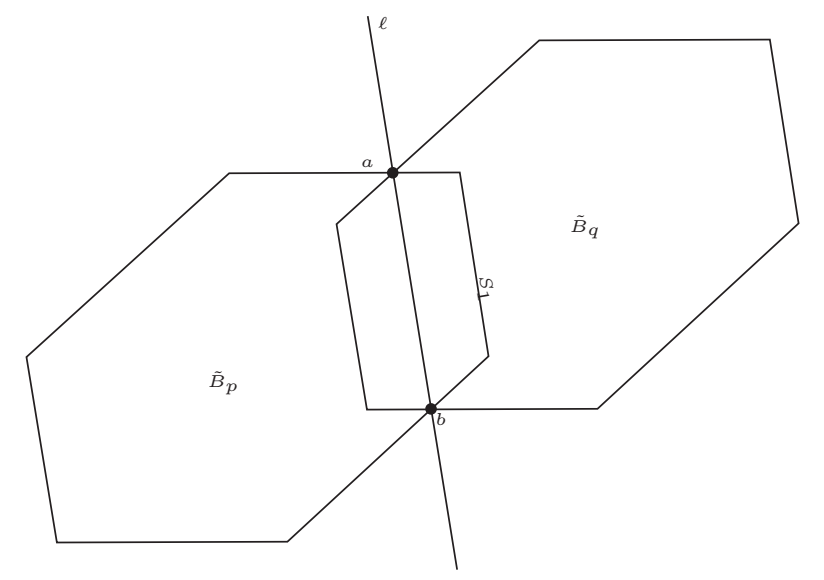

FIG. 10. $\tilde{B}_{p} \backslash \tilde{B}_{q}$ and $\tilde{B}_{q} \backslash \tilde{B}_{p}$ are separated by the line $\ell$.

Let $\ell$ denote the line through $a$ and $b$ and let us orient $\ell$ in such a way that $S_{1} \subseteq \mathbb{R}^{2} \backslash \ell^{-}$. Observe that

$$
\tilde{B}_{p} \cap\left(\mathbb{R}^{2} \backslash \ell^{-}\right)=\operatorname{conv}\left(S_{1} \cup\{a, b\}\right) \subseteq \tilde{B}_{q} .
$$

(The equality holds because $\tilde{B}_{p} \cap\left(\mathbb{R}^{2} \backslash \ell^{-}\right)$is convex and its boundary is $S_{1} \cup[a, b]$. The inclusion holds because $S_{1} \cup\{a, b\} \subseteq \tilde{B}_{q}$ and $\tilde{B}_{q}$ is convex.) This shows that $\tilde{A}_{p} \subseteq \ell^{-}$. Completely analogously, we find $\tilde{A}_{q} \subseteq \ell^{+}$. So $\ell$ is as required. This proves the statement in the case that the corner points are in general position.

Let us therefore consider the situation when the corner points are not in general position. Since $\tilde{B}$ is closed, the points of $\tilde{A}_{q}$ are all some positive distance away from $\tilde{B}_{p}$ and the points of $\tilde{A}_{p}$ are all some positive distance away from $\tilde{B}_{q}$. Thus, there exist $\lambda>1$ and $\varepsilon>0$ such that $\tilde{A}_{p} \subseteq(x+\lambda \tilde{B}) \backslash(y+\lambda \tilde{B})$ and $\tilde{A}_{q} \subseteq(y+\lambda \tilde{B}) \backslash(x+\lambda \tilde{B})$ for all $x \in B(p, \varepsilon), y \in B(q, \varepsilon)$. We can now pick $x, y$ such that the corner points of $\tilde{C}_{p}:=x+\lambda \tilde{B}$ and $\tilde{C}_{q}:=y+\lambda \tilde{B}$ are in general position and repeat the above argument for $\tilde{C}_{p}, \tilde{C}_{q}$ to find the sought line $\ell$.

Since in our original construction we had that $B+a(x, y)$ and $B+b(x, y)$ were disjoint and $W(x) \subseteq B+a(x, y), W(y) \subseteq B+B(x, y)$, whenever $x, y \in\{0, \ldots, N\}^{3}$ have Hamming distance one, the claim immediately implies

$$
\text { if } d_{\mathrm{H}}(x, y)=1, \operatorname{then} \operatorname{conv}(\tilde{W}(x)) \cap \operatorname{conv}(\tilde{W}(y))=\emptyset .
$$

Claim D-2. $\tilde{W}(x) \backslash\left\{p_{1}(x), \ldots, p_{7}(x)\right\} \subseteq \operatorname{conv}\left(\left\{\tilde{p}_{1}(x), \tilde{p}_{2}(x), \tilde{p}_{3}(x)\right\}\right)$ for all $x$.

Proof of D-2. For $i=1,2,3$, let $h_{i}$ denote an oriented line such that if $a \in$ $W(x) \cap B_{i}^{-}(x)$, then $\tilde{a} \in h_{i}^{-}$, and if $a \in W(x) \cap B_{i}^{+}(x)$, then $\tilde{a} \in h_{i}^{+}$. (By (c-1) and Claim D-1 such lines exist.) Let us also observe that by (c-1)-(c-4) and the claim

$$
\sigma\left(\tilde{p}_{i}(x) ;\left(h_{1}, h_{2}, h_{3}\right)\right)=\sigma\left(p_{i} ;\left(\ell_{1}, \ell_{2}, \ell_{3}\right)\right) \text { for } i=1, \ldots, 7 m,
$$

where $p_{i}$ and $\ell_{i}$ are as in Lemma 8. In particular we have $\tilde{p}_{i}(x) \in h_{1}^{-} \cap h_{2}^{-} \cap h_{3}^{-}$for $8 \leq i \leq 7 m$, and we can apply Lemma 7 to $\tilde{p}_{1}(x), \ldots, \tilde{p}_{7}(x)$ and $h_{1}, h_{2}, h_{3}$ to derive that $h_{1}^{-} \cap h_{2}^{-} \cap h_{3}^{-} \subseteq \operatorname{conv}\left(\left\{\tilde{p}_{1}(x), \tilde{p}_{2}(x), \tilde{p}_{3}(x)\right\}\right)$. This proves the claim.

Let us say that $1 \leq i \leq 3 m$ is bad for $x$ if $\operatorname{conv}(\tilde{W}(x))$ contains a corner of $\tilde{B}_{i}^{+}(x)$ or a corner of $\tilde{B}_{i}^{-}(x)$. We will say that $i$ is $\operatorname{good}$ for $x$ if it is not bad for $x$. 


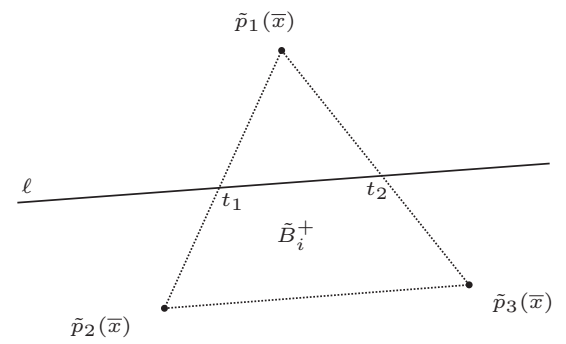

FIG. 11. The picture referred to in the proof of Claim D-3.

CLAIM D-3. If $4 \leq i \leq 3 m$ is good for $x$, then there is an oriented line $\tilde{\ell}_{i}(x)$ with slope $s \in\left\{s_{1}, \ldots, s_{k}\right\}$ such that

$$
\begin{aligned}
& \left(\tilde{W}(x) \backslash\left\{\tilde{p}_{1}(x), \ldots, p_{7}(x)\right\}\right) \cap \tilde{\ell}_{i}^{-}(x)=\left(\tilde{W}(x) \backslash\left\{\tilde{p}_{1}(x), \ldots, p_{7}(x)\right\}\right) \cap \tilde{B}_{i}^{-}(x), \\
& \left(\tilde{W}(x) \backslash\left\{\tilde{p}_{1}(x), \ldots, p_{7}(x)\right\}\right) \cap \tilde{\ell}_{i}^{+}(x)=\left(\tilde{W}(x) \backslash\left\{\tilde{p}_{1}(x), \ldots, p_{7}(x)\right\}\right) \cap \tilde{B}_{i}^{+}(x) .
\end{aligned}
$$

Proof of D-3. By (14) the points $\tilde{p}_{j}(x)$ each lie in exactly one of $\tilde{B}_{i}^{-}(x), \tilde{B}_{i}^{+}(x)$ for $j=1, \ldots, 7 m$. Recall that by Claim D-2 we have $\tilde{p}_{j}(x) \in \operatorname{conv}\left(\left\{\tilde{p}_{1}(x), \tilde{p}_{2}(x), \tilde{p}_{3}(x)\right\}\right)$ for all $8<j \leq 7 m$. Therefore, if either $\left\{\tilde{p}_{1}(x), \tilde{p}_{2}, \tilde{p}_{3}(x)\right\} \subseteq \tilde{B}_{i}^{-}(x)$ or $\left\{\tilde{p}_{1}(x), \tilde{p}_{2}(x)\right.$, $\left.\tilde{p}_{3}(x)\right\} \subseteq \tilde{B}_{i}^{+}(x)$, then it is easy to define a line $\tilde{\ell}_{i}(x)$ as required. For the remainder of the proof we can thus assume that

$$
\tilde{B}_{i}^{-}(x) \cap\left\{\tilde{p}_{1}(x), \tilde{p}_{2}(x), \tilde{p}_{3}(x)\right\}=\left\{\tilde{p}_{1}(x)\right\} .
$$

(The other cases are similar.) Let $t_{1}$ denote point of $\left[\tilde{p}_{1}(x), \tilde{p}_{2}(x)\right] \cap \tilde{B}_{i}^{+}(x)$ closest to $\tilde{p}_{1}(x)$ and let $t_{2}$ denote the point of $\left[\tilde{p}_{1}(x), \tilde{p}_{3}(x)\right] \cap \tilde{B}_{i}^{+}(x)$ closest to $\tilde{p}_{1}(x)$. Observe that $t_{1}, t_{2} \in \partial \tilde{B}_{i}^{+}(x)$. Let $\ell$ denote the line through $t_{1}$ and $t_{2}$. (See Figure 11 for a depiction.)

There can be no point of $\tilde{B}_{i}^{+}(x) \cap \operatorname{conv}\left(\left\{\tilde{p}_{1}(x), \tilde{p}_{2}(x), \tilde{p}_{3}(x)\right\}\right)$ on the same side of $\ell$ as $\tilde{p}_{1}(x)$, because otherwise a corner of $\tilde{B}_{i}^{+}(x)$ would be contained in $\operatorname{conv}(\tilde{W}(x))$. Thus, the segment $\left[t_{1}, t_{2}\right]$ is contained in $\partial B_{i}^{+}(x)$ and $\ell$ supports a side of $B_{i}^{+}(x)$. In particular $\ell$ has slope $\in\left\{s_{1}, \ldots, s_{k}\right\}$. We can orient $\ell$ such that $\tilde{B}_{i}^{+}(x) \subseteq \ell^{+} \cup \ell$. Translating $\ell$ very slightly in case some points of $\tilde{W}(x)$ lie on it, we get an oriented line that is as required.

Claim D-4. There exists an $x \in\{1, \ldots, N\}^{3}$ such that at least $2.99 \cdot m$ indices $i$ are good for $x$.

Proof of D-4. We shall first give an upper bound on the number of pairs $(i, x)$ such that $i$ is bad for $x$. We will show that

$$
\mid\{(i, x): i \text { bad for } x\} \mid \leq 3 m \cdot 4 k \cdot(N+1)^{2} .
$$

In order to prove (16), let us pick an arbitrary $1 \leq i \leq 3 \mathrm{~m}$. We can suppose that in the original line arrangement $\mathcal{L}$ of Lemma 8 the line $\ell_{i}$ has slope $s_{1}$. (The other cases are analogous.)

For each $(a, b) \in\{0, \ldots, N\}^{2}$, let us set

$$
X(a, b):=\left\{x \in\{0, \ldots, N\}^{3}: x_{2}=a, x_{3}=b\right\} .
$$



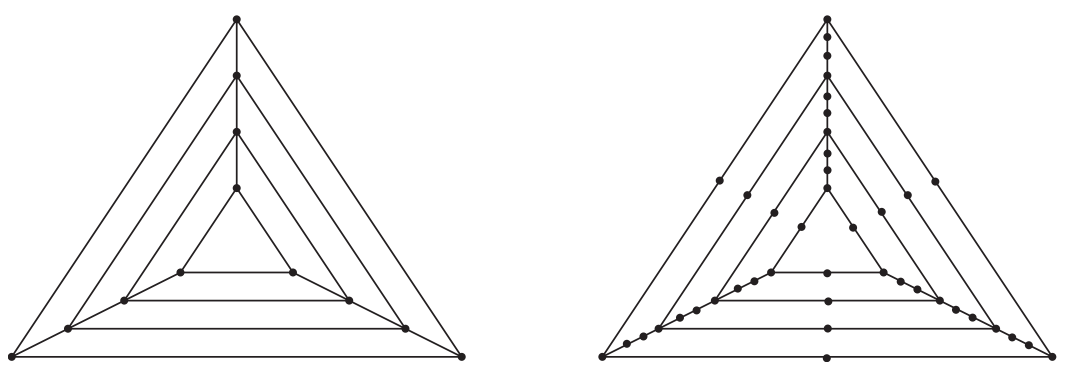

FIG. 12. The triangular prism $T_{4}$ of height 4 and the graph $T_{2,4}$.

Observe that the sets $X(a, b)$ partition $\{0, \ldots, N\}^{3}$. Recall that, by (c-4) (and also because $\mathcal{V}$ was an ordinary set, not a multiset), $\tilde{c}_{i}^{+}(x)=\tilde{c}_{i}^{+}(y)$ and $\tilde{c}_{i}^{-}(x)=\tilde{c}_{i}^{-}(y)$ for all $x, y \in X(a, b)$. Since $\tilde{B}_{i}^{-}(x)$ and $\tilde{B}_{i}^{+}(x)$ both have $2 k$ corners, and the sets $\operatorname{conv}(\tilde{W}(x)): x \in X(a, b)$ are disjoint by (15), we see that $i$ is bad for at most $4 k$ elements of $X(a, b)$. This gives that $i$ is bad for at most $4 k \cdot(N+1)^{2}$ of all $x \in\{0, \ldots, N+1\}^{3}$. Since $i$ was arbitrary, we get (16).

Because there are a total of $(N+1)^{3}$ choices of $x$, there is some $x$ for which at most

$$
\frac{3 m \cdot 4 k \cdot(N+1)^{2}}{(N+1)^{3}}=\frac{3 m \cdot 4 k}{N+1}<0.01 m
$$

indices $i$ are bad (recall that $N=10^{10} \cdot k$ ). This proves the claim.

Now let $x$ be as provided by Claim D-4. Let $A \subseteq\{1, \ldots, 3 m\}$ denote the indices that are good for $x$. For $i \in A$, let $\tilde{\ell}_{i}(x)$ be as provided by Claim D-3, and for $i \notin A$ let $\tilde{\ell}_{i}(x)$ be an arbitrary line with slope $\in\left\{s_{1}, \ldots, s_{k}\right\}$. Let us set $B:=\{8, \ldots, 7 m\}$. Then $A, B, \tilde{\mathcal{L}}:=\left(\tilde{\ell}_{1}(x), \ldots, \tilde{\ell}_{3 m}(x)\right)$ and $\tilde{W}(x)$ satisfy the conditions of part (iii) of Lemma 8. Thus, there are distinct $p, q, r, s \in \tilde{W}(x)$ such that

$$
\|p-q\| \geq \alpha^{m}\|r-s\| \geq \alpha^{m} .
$$

Here we used that $r-s$ is exactly the difference vector between a corner of $r+\tilde{P}$ and a corner of $s+\tilde{P}$, so that $r-s \in \mathbb{Z}^{2}$ and hence $\|r-s\| \geq 1$. Hence if all corner points are contained in $[-K, K]^{2}$, then we must have $K \geq \alpha^{m} / 2 \sqrt{2}=2^{\Omega(m)}$. Since $\left|V\left(G_{m, N}\right)\right|=O(m)$ this concludes the proof.

5. Lower bound for $\boldsymbol{P}$-homothet graphs. In this section, we will prove the following result: let $P$ be an arbitrary polygon with rational corner points, then $h_{P}(n)=2^{\Omega(n)}$. We begin with the construction of a hard family of $P$-homothet graphs.

For $m \in \mathbb{N}$, let $T_{m}$ denote the triangular prism of height $m$. That is, $T_{m}$ is the graph we obtain if we take $m$ vertex-disjoint triangles $C_{1}, \ldots, C_{m}$ and we add matchings between $V\left(C_{i}\right)$ and $V\left(C_{i+1}\right)$ for all $i=1, \ldots, m-1$. For $k, m \in \mathbb{N}$ let $T_{k, m}$ denote the graph that is obtained from $T_{m}$ by subdividing the edges of the triangles once and subdividing the edges of the matchings $k$ times. (See Figure 12.)

We will rely on the following observation. (See Figure 13.)

Lemma 9. Let $P$ be an arbitrary polygon. Then there is a $k=k(P)$ such that $T_{k, m}$ is a P-homothets graph for all $m \geq 1$.

We leave the straightforward proof of Lemma 9 to the reader. To prove that $T_{k, m}$ needs a large grid size, we will first need to prove a number of intermediate results. 


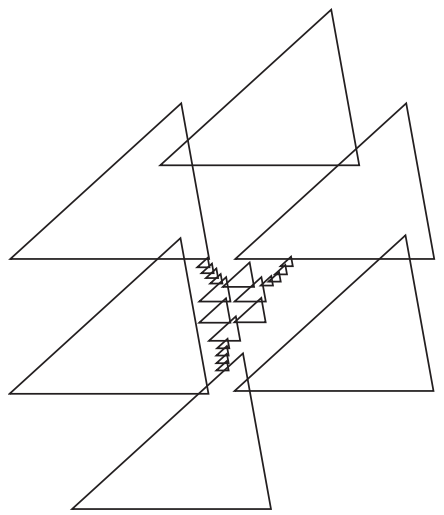

FIG. 13. A P-homothets representation of the graph $T_{4,2}$.

Recall that a planar embedding (or drawing) of a planar graph $G$ is a set of distinct points $\{p(v): v \in V(G)\}$ in the plane, together with simple (i.e., not self-intersecting) curves $\{c(e): e \in E(G)\}$ such that $c(u v)$ has endpoints $p(u), p(v)$ and any two of the curves $c(e), c(f)$ do not intersect except possibly at a common endpoint (cf. [23]).

Lemma 10. Suppose that $\mathcal{A}=\left\{A(v): v \in T_{k, m}\right\}$ is a family of closed convex sets in the plane, each with nonempty interior, that realize $T_{k, m}$ as their intersection graph. Then there exists a planar embedding of $T_{k, m}$ with $p(v) \in A(v)$ for every $v \in V\left(T_{k, m}\right)$ and $c(u v) \subseteq A(u) \cup A(v)$ for every edge $u v \in E\left(T_{k, m}\right)$.

Proof. We shall first define sets $B(v)$ such that $B(v)=A(v)$ if $d(v)=3$ and $B(v) \subseteq A(v)$ is a line segment when $d(v)=2$, and moreover

$$
|B(u) \cap B(v)|= \begin{cases}1 & \text { if } u v \in E\left(T_{k, m}\right), \\ 0 & \text { otherwise. }\end{cases}
$$

Order the vertices as $v_{1}, \ldots, v_{n}$ arbitrarily. We shall define the sets $B(v)=B^{(n)}(v)$ by means of the following procedure. We set $B^{(0)}(v)=A(v)$ for all $v \in V\left(T_{k, m}\right)$. At step $1 \leq i \leq n$, we consider the vertex $v_{i}$. If $d\left(v_{i}\right)=3$, then we set $B^{(i)}(v)=B^{(i-1)}(v)$ for all $v$. If $d\left(v_{i}\right)=2$, then we do the following. Write $N\left(v_{i}\right)=\{u, w\}$. We pick points $a \in B^{(i-1)}\left(v_{i}\right) \cap B^{(i-1)}(u), b \in B^{(i-1)}\left(v_{i}\right) \cap B^{(i-1)}(w)$ of smallest possible distance, and we put $B^{(i)}\left(v_{i}\right):=[a, b]$ and $B^{(i)}(v)=B^{(i-1)}(v)$ for all other $v$. A straightforward inductive argument shows that the sets $B(v)$ satisfy (17).

For $u v \in E\left(T_{k, m}\right)$, let $q(u v)$ be the unique point of $B(u) \cap B(v)$. For $v \in V\left(T_{k, m}\right)$ with degree $d(v)=2$ we define a point $p(v)$ to be the midpoint of the segment $[q(u v), q(w v)] \subseteq B(v)$, where $u, w$ are the two neighbors of $v$. For $d(v)=3$ we do the following. Write $N(v)=\{u, w, s\}$ and $B(v) \cap B(u)=\{a\}, B(v) \cap B(w)=$ $\{b\}, B(v) \cap B(s)=\{c\}$. Observe that $a, b, c \in \partial A(v)$ (recall $B(v)=A(v)$ ), since $B(u), B(w), B(s)$ are segments that intersect $A(v)$ in exactly one point. Let $\ell_{1}, \ell_{2}, \ell_{3}$ denote the lines through $a, b$, resp., $a, c$, resp., $b, c$. We now pick an arbitrary $p(v) \in$ $\operatorname{int}(A(v)) \backslash\left(\ell_{1} \cup \ell_{2} \cup \ell_{3}\right)$

By construction, for every two edges $u v, s t \in E\left(T_{k, m}\right)$,

$$
[p(v), q(u v)] \cap[p(s), q(s t)]=\left\{\begin{array}{cl}
{[p(v), q(u v)]} & \text { if } v=s, u=t \\
\{p(v)\} & \text { if } v=s, u \neq t \\
\{q(u v)\} & \text { if } v=t, u=s \\
\emptyset & \text { otherwise }
\end{array}\right.
$$

Copyright $@$ by SIAM. Unauthorized reproduction of this article is prohibited. 
(To see this recall that $[p(v), q(u v)] \subseteq B(v),[p(s), q(s t)] \subseteq B(s)$, so that $[p(v), q(u v)] \cap$ $[p(s), q(s t)] \neq \emptyset$ implies that either $v=s$ or $[p(v), q(u v)] \cap[p(s), q(s t)]=\{q(v s)\}$. The statement (18) now follows from the fact that the segments $[p(v), q(v w)]$ with $w \in N(v)$ intersect only in $p(v)$ by construction.)

Finally, let us observe that the required embedding can be defined by setting $c(u v):=[p(v), q(u v)] \cup[q(u v), p(u)]$ for every edge $u v \in E\left(T_{k, m}\right)$. That this is a proper embedding follows immediately from (18).

Recall that by the Jordan curve theorem, a simple closed curve $c$ divides $\mathbb{R}^{2} \backslash c$ into two components. The bounded one is called the inside of $c$, and we shall denote it by inside $(c)$.

If $c_{1}, \ldots, c_{k}$ are simple closed curves in the plane, then we say that they are nested if either $c_{i} \subseteq \operatorname{inside}\left(c_{i+1}\right)$ for all $i=1, \ldots, k-1$ or $c_{i+1} \subseteq \operatorname{inside}\left(c_{i}\right)$ for all $i=1, \ldots, k-1$. We need the following straightforward observation, whose proof we leave to the reader.

Lemma 11. Let $p(v): v \in V\left(T_{k, m}\right)$ and $c(e): e \in E\left(T_{k, m}\right)$ be a planar embedding of $T_{k, m}$. Let $\gamma_{1}, \ldots, \gamma_{m}$ be the closed curves corresponding to the $m$ six-cycles of $T_{k, m}$. Then either $\gamma_{1}, \ldots, \gamma_{\lfloor m / 2\rfloor}$ are nested, or $\gamma_{\lfloor m / 2\rfloor+1}, \ldots, \gamma_{m}$ are nested.

We now proceed towards the lower bound argument.

Lemma 12. For all $k \in \mathbb{N}$ and $c>0$ there exists an $\alpha=\alpha(k, c)>1$ and an $m_{0}=$ $m_{0}(k, c) \in \mathbb{N}$ such that the following holds. If $m \geq m_{0}$ and $\mathcal{A}=\left\{A(v): v \in V\left(T_{k, m}\right)\right\}$ is a collection of subsets of the plane with the properties

(i) the intersection graph of $\mathcal{A}$ is isomorphic to $T_{k, m}$;

(ii) every $A \in \mathcal{A}$ is closed, convex and has nonempty interior;

(iii) $\operatorname{area}(A) \geq c \cdot \operatorname{diam}^{2}(A)$ for all $A \in \mathcal{A}$, then there exist $A, A^{\prime} \in \mathcal{A}$ such that $\operatorname{diam}(A) \geq \alpha^{m} \cdot \operatorname{diam}\left(A^{\prime}\right)$.

Proof. Let $C_{1}, \ldots, C_{m}$ denote the $m$ six-cycles of $T_{k, m}$. Let $c_{1}, \ldots, c_{m}$ be the corresponding closed curves in the embedding provided by Lemma 10 , and let $v_{1, i}, \ldots, v_{6, i}$ denote the vertices of $C_{i}$. We can assume without loss of generality that the numbering is such that $\operatorname{diam}\left(A\left(v_{1, i}\right)\right) \geq \operatorname{diam}\left(A\left(v_{2, i}\right)\right), \ldots, \operatorname{diam}\left(A\left(v_{6, i}\right)\right)$ for all $i=1, \ldots, m$. Appealing to Lemma 11 , we can assume without loss of generality that $c_{i+1} \subseteq \operatorname{inside}\left(c_{i}\right)$ for $i=1, \ldots,\lfloor m / 2\rfloor-1$.

Since $c_{i} \subseteq A\left(v_{1, i}\right) \cup \cdots \cup A\left(v_{6, i}\right)$ we have

$$
\operatorname{diam}\left(c_{i}\right) \leq 6 \cdot \operatorname{diam}\left(A\left(v_{1, i}\right)\right) .
$$

By the isodiametric inequality, we must have

$$
\text { area(inside } \left.\left(c_{i}\right)\right) \leq \frac{\pi}{4}\left(6 \operatorname{diam}\left(A\left(v_{1, i}\right)\right)\right)^{2}=9 \pi \operatorname{diam}^{2}\left(A\left(v_{1, i}\right)\right) .
$$

Now let $K$ be defined as

$$
K:=\lceil 1000 / c\rceil .
$$

We claim that there must be a sequence $i(0):=1<i(1)<\cdots<i(\lfloor m / K\rfloor) \leq m$ such that $\operatorname{diam}\left(A\left(v_{1, i(j+1)}\right)\right) \leq \frac{1}{2} \operatorname{diam}\left(A\left(v_{1, i(j)}\right)\right)$ for $j=0, \ldots,\lfloor m / K\rfloor-1$. To see this, suppose that for some $j \geq 1$ we have already defined $i(0), \ldots, i(j-1)$. Suppose that $\operatorname{diam}\left(A\left(v_{1, i(j-1)+1}\right)\right), \ldots, \operatorname{diam}\left(A\left(v_{1, i(j-1)+K}\right)\right)>\frac{1}{2} \operatorname{diam}\left(A\left(v_{1, i(j-1)}\right)\right)$. Then we have

$$
\begin{aligned}
\operatorname{area}\left(\bigcup_{z=1}^{K} A\left(v_{1, i(j-1)+z}\right)\right) & \geq K \cdot \frac{1}{2} \cdot c \cdot \operatorname{diam}^{2}\left(A\left(v_{1, i(j-1)}\right)\right) \\
& \geq 500 \operatorname{diam}^{2}\left(A\left(v_{1, i(j-1)}\right)\right) \\
& >9 \pi \operatorname{diam}^{2}\left(A\left(v_{1, i(j-1)}\right)\right) \\
& \geq \operatorname{area}\left(\operatorname{inside}\left(c_{i(j-1)}\right)\right)
\end{aligned}
$$

Copyright $@$ by SIAM. Unauthorized reproduction of this article is prohibited. 
using that the sets $A\left(v_{1, i}\right): i=1, \ldots, m$ are disjoint (as the corresponding vertices are nonadjacent in $\left.T_{k, m}\right)$ in the first line.

On the other hand, since $c_{i(j-1)+1}, \ldots, c_{i(j-1)+K} \subseteq \operatorname{inside}\left(c_{i(j-1)}\right)$ and $A\left(v_{1, i(j-1)+z}\right)$ does not intersect $A\left(v_{1, i(j-1)}\right) \cup \cdots \cup A\left(v_{6, i(j-1)}\right)$ for all $z \geq 1$, we must have

$$
A\left(v_{1, i(j-1)+1}\right), \ldots, A\left(v_{1, i(j-1)+K}\right) \subseteq \operatorname{inside}\left(c_{i(j-1)}\right) .
$$

This implies that

$$
\text { area }\left(A\left(v_{i(j-1)+1}\right) \cup \cdots \cup A\left(v_{i(j-1)+K}\right)\right) \leq \operatorname{area}\left(\operatorname{inside}\left(c_{i(j-1)}\right)\right),
$$

which contradicts (21).

It follows that one of $\operatorname{diam}\left(A\left(v_{1, i(j-1)+1}\right)\right), \ldots, \operatorname{diam}\left(A\left(v_{1, i(j-1)+K}\right)\right)$ is at most $\frac{1}{2} \operatorname{diam}\left(A\left(v_{1, i(j-1)}\right)\right)$. Thus, we can pick $i(j-1)<i(j) \leq i(j-1)+K$ such that $\operatorname{diam}\left(A\left(v_{1, i(j)}\right)\right) \leq \frac{1}{2} \operatorname{diam}\left(A\left(v_{1, i(j-1)}\right)\right)$. This proves the claim.

To finish the proof of the theorem, note that

$$
\operatorname{diam}\left(A\left(v_{1,1}\right)\right) / \operatorname{diam}\left(A\left(v_{1, i(\lfloor m / K\rfloor)}\right)\right) \geq 2^{\lfloor m / K\rfloor} \geq \alpha^{m}
$$

for $m$ sufficiently large, where we can, for instance, take $\alpha:=\sqrt[2 K]{2}$.

THEOREM 7. Let $P$ be an arbitrary convex polygon with rational corner points. Then $h_{P}(n)=2^{\Omega(n)}$.

Proof. By Lemma 9, there is a $k=k(P)$ such that $T_{k, m}$ is a $P$-homothets graph for all $m \in \mathbb{N}$.

Now let $m \in \mathbb{N}$ be arbitrary and let $\mathcal{A}=\left\{A(v): v \in V\left(T_{k, m}\right)\right\}$ be a collection of homothets of $P$, whose intersection graph is exactly $T_{k, m}$ and all of whose corner points lie on $\mathbb{Z}^{2}$. Let us write $n:=\left|V\left(T_{k, m}\right)\right|$. Observe that $n=\Theta(m)$.

Since each $A \in \mathcal{A}$ is a $P$-homothet, we have

$$
\operatorname{area}(A) \geq c \cdot \operatorname{diam}^{2}(A) \quad \text { for all } A \in \mathcal{A}
$$

with $c:=\operatorname{area}(P) / \operatorname{diam}^{2}(P)$. Hence we can apply Lemma 12. Thus, there are $A, A^{\prime} \in \mathcal{A}$ with $\operatorname{diam}(A) \geq \alpha^{m} \operatorname{diam}\left(A^{\prime}\right)$ with $\alpha=\alpha(P)>1$ a constant that depends only on $P$. Since the corner points of $A^{\prime}$ lie on the grid, we must have $\operatorname{diam}\left(A^{\prime}\right) \geq 1$. Let $p, q$ be the corner points of $A$ that realize its diameter. Then

$$
\|p-q\| \geq \alpha^{m} .
$$

This shows that if $p, q$ both lie inside a $[-K, K]^{2}$, then we must have $K \geq \alpha^{m} / 2 \sqrt{2}=$ $2^{\Omega(n)}$.

6. Conclusions and further work. We determined precise upper and lower bounds on the grid size needed for representing convex polygon intersection graphs, depending on the polygon $P$ and the type of similarity transforms allowed (translations or homothetic transforms). The results imply a quadratic bound for parallelogramtranslate graphs and a surprising "singly exponential" bound in all other cases.

Several questions remain open. A number of them relate to the complexity of recognizing $\operatorname{trans}(P)$ and $\operatorname{hom}(P)$ graphs. As in [31], our results imply that both recognition problems are in $\mathrm{NP}$, on the assumption that $P$ has rational corner points. This, however, does not settle the problem for polygons $P$ that do not have rational corner points. In this case, $P$ may not be representable in finitely many bits and the grid model for representing intersection graphs seems to fail. Nevertheless, are 
the trans $(P)$-recognition and hom $(P)$-recognition problems perhaps still in NP for all convex polygons $P$ ? (Here we think of $P$ as implicit, i.e., not given as part of the input.)

The NP-hardness issue for the recognition problems is of interest as well. For $\operatorname{hom}(P)$ the recognition problem was shown to be NP-hard $[24,17]$ for all convex polygons $P$, but we are not aware of any result for the recognition problem for the class trans $(P)$, other than for the case when $P$ is the unit square. For this case Breu [3] proved the recognition problem to be NP-hard. Can the proofs of [3] or [17] be modified to show that the recognition problem for $\operatorname{trans}(P)$ is NP-hard for all convex polygons $P$ as well?

If $P$ itself does not have rational corner points, then one might try to find a polygon $Q$ similar to $P$ that does. Note that by Lemma 1 our results also apply if $P$ can be transformed into a rational polygon by means of an affine transformation. (This is, for instance, the case for all triangles). Hence, a naive approach to settle the problems above might be to find a rational polygon $Q$ such that $\operatorname{trans}(P)=\operatorname{trans}(Q)$, resp., $\operatorname{hom}(P)=\operatorname{hom}(Q)$, for every $P$ that does not have rational corner points. However, we strongly suspect that this is not possible. The reason is that we suspect that Lemma 1 can be turned into an if-and-only-if statement. In other words, that $\operatorname{trans}(P)=\operatorname{trans}(Q)$ if and only if $P=T[Q]$ for some affine transformation $T$ and that $\operatorname{hom}(P)=\operatorname{hom}(Q)$ if and only if $P=T[Q]$ for some affine transformation $T$. A result of Cerný et al. [5, Theorem 1] suggests that our suspicion may be right, but the fact remains to be proved.

Finally, another open question is the generalization to higher dimensions. As mentioned in section 2, the upper bounds extend trivially to higher dimensions. However, it is not clear how much of the lower bound constructions can be salvaged in dimension $d \geq 3$.

Acknowledgment. We thank the referees for helpful suggestions which have improved the presentation.

\section{REFERENCES}

[1] P. K. Agarwal, J. PaCh, AND M. Sharir, State of the union (of geometric objects), in Surveys on Discrete and Computational Geometry, Contemp. Math. 453, AMS, Providence, RI, 2008, pp. 9-48.

[2] S. Arora And B. Barak, Computational Complexity: A Modern Approach, Cambridge University Press, Cambridge, UK, 2009.

[3] H. Breu, Algorithmic Aspects of Constrained Unit Disk Graphs, Ph.D. thesis, University of British Columbia, Vancouver, 1996.

[4] H. Breu and D. G. Kirkpatrick, Unit disk graph recognition is NP-hard, Comput. Geom., 9 (1998), pp. 3-24.

[5] J. Černý, D. Král, H. Nyklová, and O. Pangrác, On intersection graphs of segments with prescribed slopes, in Graph Drawing 2001, Proceedings, S. G. Kobourov and M. T. Goodrich, eds., Lecture Notes in Comput. Sci. 2265, Springer, Berlin, 2002, pp. 261-271.

[6] D. G. Corneil, H. Kim, S. Natarajan, S. Olariu, and A. P. Sprague, Simple linear time recognition of unit interval graphs, Inform. Process. Lett., 55 (1995), pp. 99-104.

[7] J. Czyzowicz, E. Kranakis, D. Krizanc, and J. Urrutia, Discrete realizations of contact and intersection graphs, Int. J. Pure Appl. Math., 13 (2004), pp. 429-442.

[8] H. de Fraysseix, J. Pach, and R. Pollack, How to draw a planar graph on a grid, Combinatorica, 10 (1990), pp. 41-51.

[9] G. Ehrlich, S. Even, and R. E. Tarjan, Intersection graphs of curves in the plane, J. Combinatorial Theory Ser. B, 21 (1976), pp. 8-20.

[10] M. C. Golumbic, Algorithmic Graph Theory and Perfect Graphs, Academic Press, New York, 1980. 
[11] M. C. Golumbic And A. N. Trenk, Tolerance Graphs, Cambridge University Press, Cambridge, UK, 2004.

[12] R. J. Kang And T. Müller, Sphere and dot product representations of graphs, Discrete Comput. Geom., 47 (2012), pp. 548-568.

[13] M. Kaufmann, J. Kratochvíl, K. A. Lehmann, and A. R. Subramanian, Max-tolerance graphs as intersection graphs: Cliques, cycles, and recognition, in Proceedings of the 17th Annual ACM-SIAM Symposium on Algorithms (SODA 2006), ACM Press, New York, 2006, pp. 832-841.

[14] J. KRatochvíl, String graphs II: Recognizing string graphs is NP-hard, J. Combin. Theory Ser. B, 52 (1991), pp. 67-78.

[15] J. Kratochvíl And J. MatoušEK, String graphs requiring exponential representations, J. Combin. Theory Ser. B, 53 (1991), pp. 1-4.

[16] J. Kratochvíl and J. MatoušEK, Intersection graphs of segments, J. Combin. Theory Ser. B, 62 (1994), pp. 289-315.

[17] J. Kratochvíl And M. Pergel, Intersection graphs of homothetic polygons, in International Conference on Topological and Geometric Graph Theory, Electron. Notes Discrete Math. 31, Elsevier Science, Amsterdam, 2008, pp. 277-280.

[18] J. MatoušEk, Lectures on Discrete Geometry, Grad. Texts in Math. 212, Springer-Verlag, New York, 2002.

[19] C. J. H. MCDiarmid And T. MüLler, Integer representations of disk and segment graphs, J. Combin. Theory Ser. B, 103 (2013), pp. 114-143.

[20] T. McKeE And F. McMorRIs, Topics in Intersection Graph Theory, SIAM Monogr. Discrete Math. Appl. 2, SIAM, Philadelphia, 1999.

[21] G. B. Mertzios, The recognition of triangle graphs, Theoret. Comput. Sci., 438 (2012), pp. 3447.

[22] G. B. Mertzios And D. G. Corneil, Vertex splitting and the recognition of trapezoid graphs, Discrete Appl. Math., 159 (2011), pp. 1131-1147.

[23] T. Nishizeki and M. S. Rahman, Planar Graph Drawing, Lecture Notes Ser. Comput. 12, World Scientific, Hackensack, NJ, 2004.

[24] M. Pergel, Special Graph Classes and Algorithms on Them, Ph.D. thesis, Department of Applied Mathematics, Charles University, Prague, 2008; also available online from http://kam.mff.cuni.cz/ perm/main.pdf.

[25] F. S. Roberts, On the boxicity and cubicity of a graph, in Recent Progress in Combinatorics, Proceedings of the Third Waterloo Conference on Combinatorics, Academic Press, New York, 1969, pp. 301-310.

[26] M. SchaEfer, Complexity of some geometric and topological problems, in Graph Drawing 2009, Proceedings, D. Eppstein and E. R. Gansner, eds., Lecture Notes in Comput. Sci. 5849, Springer-Verlag, New York, 2010.

[27] W. SCHNYDER, Embedding planar graphs on the grid, in Proceedings of SODA, 1990, pp. 138148.

[28] A. SchriJver, Theory of Linear and Integer Programming, Wiley-Intersci. Ser. Discrete Math.. John Wiley \& Sons, Chichester, UK, 1986.

[29] J. P. Spinrad, Efficient Graph Representations, Fields Inst. Monogr. 19, AMS, Providence, RI, 2003.

[30] E. J. Van Leeuwen, Optimization and Approximation on Systems of Geometric Objects, Ph.D. thesis, University of Amsterdam, 2009; also available online from http://dare.uva. $\mathrm{nl} /$ record/307611.

[31] E. J. van Leeuwen and J. VAn Leeuwen, Convex polygon intersection graphs, in Graph Drawing 2010, Proceedings, U. Brandes and S. Cornelsen, eds., Lecture Notes in Comput. Sci. 6205, Springer-Verlag, New York, 2010, pp. 377-388.

Copyright (c) by SIAM. Unauthorized reproduction of this article is prohibited. 